\title{
Composición de las capturas y descartes generados en la pesca de merluza común Merluccius hubbsi y langostino patagónico Pleoticus muelleri: un caso de estudio en la flota fresquera de altura del Golfo San Jorge, Chubut, Argentina
}

Catches composition and discards generated by hake Merluccius hubbsi and shrimp Pleoticus muelleri fisheries: a case of study in the high-sea ice trawlers of San Jorge Gulf, Chubut, Argentina

\author{
Nelson D. Bovcon ${ }^{1}$, María E. Góngora ${ }^{1,2}$, Cristian Marinao ${ }^{1,3}$ \\ y Diego González-Zevallos ${ }^{3}$
}

\author{
${ }^{1}$ Facultad de Ciencias Naturales, Universidad Nacional de la Patagonia San Juan Bosco, Sede Trelew, Julio A. Roca 115, 1으 \\ piso, Trelew, Chubut, CP 9100, Argentina.nelsonbovcon@hotmail.com \\ ${ }^{2}$ Secretaría de Pesca de la Provincia del Chubut, Vachina 164, CP 9103, Argentina \\ ${ }^{3}$ Centro Nacional Patagónico (CONICET), Blvd. Brown 2915, U9120ACD, Puerto Madryn, Chubut, Argentina
}

\begin{abstract}
The bottom trawling is the word most used fishing art generating large amounts of discards and incidental captures with a wide variety of species. In the San Jorge Gulf Argentine Patagonia, operates a fleet whose target specie is the hake Merluccius hubbsi, alternating with red shrimp Pleoticus muelleri. The information for this study covers a period of 10 years (2003-2012) and was collected by the On-board Observer Program of the Chubut Province (POBCh). Were identified a total of 90 taxa, 33 invertebrates, 34 osteichthyes and 22 chondrichthyes. Multivariate analysis indicated differences in the composition of the catch when the target species was shrimp or hake. When the target species was the hake catch composition varied by fishing areas, this pattern was not observed when the target species was the shrimp, with different numbers of taxa in the northern and central Gulf (north: hake 79 taxa, shrimp 71, center: hake 60 taxa, shrimp 50). The utilization of different species caught when the fleet operated on hake was integral, however when the fleet operated on shrimp most of the taxa were discarded. This study characterized the composition of the fleet catches at San Jorge Gulf, described the species caught when the fleet operates on hake or shrimp, analyzes the frequency of occurrence and abundance and describes the use of different taxa identified.
\end{abstract}

Key words: Catches composition, Merluccius hubbsi, Pleoticus muelleri, ice-trawlers fleet, San Jorge Gulf

\begin{abstract}
Resumen.- La pesca de arrastre de fondo es el arte más utilizado a nivel mundial generando grandes cantidades de descarte por la captura incidental de una amplia variedad de especies. En el Golfo San Jorge en la Patagonia Argentina, opera una flota fresquera de altura cuya especie objetivo es la merluza Merluccius hubbsi, alternando con mareas a langostino Pleoticus muelleri. La información para el presente estudio abarca un periodo de 10 años (2003-2012) y fue obtenida por el Programa de Observadores a Bordo de la Provincia del Chubut (POBCh). Se identificaron un total de 90 taxones, 33 invertebrados, 34 peces óseos y 22 peces cartilaginosos. Los análisis multivariados describen 2 agrupamientos, el primero cuando la especie objetivo fue la merluza y el otro cuando la especie objetivo fue langostino. Cuando la especie objetivo fue la merluza la composición de las capturas varió según las áreas de pesca aunque no se observó este patrón cuando la especie objetivo fue el langostino, con distintos números de taxones el norte y el centro del golfo (norte: merluza 79 taxones, langostino 71; centro: merluza 60 taxones, langostino 50). En la flota fresquera de altura, el aprovechamiento de las especies capturadas fue integral cuando la pesca estuvo dirigida a merluza, en cambio cuando el objetivo fue el langostino se descartaron la mayoría de los taxones. El presente trabajo caracteriza la composición de las capturas en la flota fresquera de altura del Golfo San Jorge, describe las especies capturadas cuando el esfuerzo estuvo dirigido a merluza o langostino, analiza las frecuencias de ocurrencia y abundancias y describe el aprovechamiento de los distintos taxones identificados.
\end{abstract}

Palabras clave: Composición de las capturas, Merluccius hubbsi, Pleoticus muelleri, flota fresquera, Golfo San Jorge

\section{INTRODUCCIÓN}

En más de 25 países, la mayoría de los desembarques de las pesquerías de arrastre de peces demersales se componen de especies pertenecientes a la familia
Merlucciidae. Argentina lidera los desembarques de esta familia con la captura de merluza común Merluccius hubbsi Marini, 1933 seguida por pesquerías de Chile, Perú, 
Sudáfrica y Estados Unidos (Kelleher 2008). La pesca en la Argentina se estructuró sobre esta especie, la cual se distribuye sobre la plataforma continental de Argentina y Uruguay (Bezzi \& Dato 1995, Bezzi et al. 2004).

En el Golfo San Jorge, se desarrolla una pesquería industrial de merluza común junto con una pesquería industrial de langostino patagónico (Pleoticus muelleri Bate, 1888). En estas pesquerías participan 2 estratos de flotas, una flota congeladora tangonera que opera exclusivamente dirigida a langostino y una flota fresquera de altura que opera tradicionalmente dirigida a merluza pero que incursiona en la pesquería de langostino patagónico (Góngora et al. 2012). Desde el desarrollo de la pesquería de langostino a fines de los 80 y principios de los 90, ambas pesquerías se solapan espacialmente y en menor medida temporalmente, en ambas pesquerías se descarta merluza (Dato et al. 2006, Góngora et al. 2012). El descarte de merluza en la pesquería de langostino es el principal problema de manejo que enfrenta esta pesquería, ya que descarta merluza en sus principales áreas de cría: el Golfo San Jorge y el Área de Veda de Juveniles de Merluza (Pettovello 1999, Bezzi et al. 2004). Esta especie es el recurso base de las flotas fresqueras del país y es un recurso actualmente en crisis (Cordo 2006, Renzi et al. 2009).

A nivel mundial resulta difícil determinar los descartes por especie o grupos de especies ya que la composición de los descartes a menudo es registrada de manera inadecuada (Kelleher 2008). La mayoría de los estudios se enfocan sobre los descartes de especies comerciales o especies en peligro, quedando fuera de los análisis muchas especies sin importancia comercial. Por otro lado, los estudios de caracterización de la fauna acompañante que incluyen además el destino final de las especies capturadas, aportan un enfoque más efectivo para la investigación de mercados y productos orientados a facilitar una mayor utilización de esas especies, a la vez que contribuyen en estudios sobre biodiversidad, manejo e impacto de la pesca en los ecosistemas marinos (Kelleher 2008). El presente trabajo caracterizó la composición de las capturas en la flota fresquera de altura del Golfo San Jorge, con la descripción de las especies capturadas cuando la flota en su operación estuvo dirigida a la merluza o langostino, analizando las frecuencias de ocurrencia y abundancias, y el análisis del aprovechamiento en los distintos taxones identificados con el fin de encontrar diferencias entre las distintas operatorias de pesca.

\section{Materiales y MÉtodos}

El Golfo San Jorge se extiende desde Cabo Dos Bahías

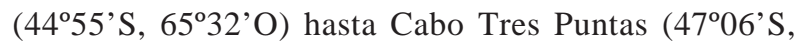
$65^{\circ} 52^{\prime} \mathrm{O}$ ), cubriendo una superficie de más de $32.270 \mathrm{~km}^{2}$. Conforma una unidad biológica y productiva administrada por las Provincias del Chubut y Santa Cruz donde funcionan 3 puertos: Caleta Córdova y Comodoro Rivadavia en la Provincia del Chubut, y Caleta Paula en la Provincia de Santa Cruz. El Golfo San Jorge tiene 2 áreas de veda permanentes, una en el norte para proteger la reproducción del langostino, en la zona de las Islas Robredo desde 2006 y otra en el sur que protege la principal área de crianza de langostino en Bajo Mazarredo desde 1985 (Fig. 1).

La flota bajo estudio está compuesta aproximadamente por 15 buques fresqueros de altura. Esta flota opera en aguas del Golfo San Jorge, principalmente en la jurisdicción de la Provincia de Chubut. Las embarcaciones pertenecientes a esta flota poseen casco de acero con una eslora de 26,4 $\pm 2,4 \mathrm{~m}$ (rango = 21,2-30,9 m), una manga de $6,4 \pm 0,2 \mathrm{~m}$ (rango $=6,1-7,0 \mathrm{~m}$ ) y una potencia de motor de 458,1 $\pm 65,0$ HP (rango $=380-624$ HP). La capacidad de bodega es de 145,1 $\pm 28,8 \mathrm{~m}^{3}$ (rango $=90-200 \mathrm{~m}^{3}$ ), estimándose unos 11 cajones por $\mathrm{m}^{3}$. Desde el año 2000 la mayoría de los buques pesqueros que componen la flota fresquera de altura del Golfo San Jorge incorporaron tangones a sus embarcaciones, alternando mareas dirigidas a merluza o a langostino.

Para analizar la composición de las capturas se utilizó la información recolectada en la flota fresquera de altura por el Programa de Observadores a Bordo perteneciente a la Provincia del Chubut (POBCh). Los observadores a bordo registraron en cada lance de pesca las especies capturadas junto a la especie objetivo. De cada especie se consignó la abundancia en número en 4 categorías y su destino. Las categorías utilizadas en la estimación de la abundancia fueron las definidas por el POBCh: dominante (Do: la especie representa más del 50\% de la captura en número, su presencia da el aspecto general de la captura), abundante (Ab: entre 25 y 50\%, se observa con facilidad su presencia), común (Co: entre 5 y 25\%, se observa al prestar atención y revolver las capturas) y rara (Ra: menos del 5\%, pocos individuos). El destino de la especie puede ser: totalmente encajonada (En: encajonada), parcialmente encajonada (Pe: parcialmente encajonada) o totalmente arrojada al mar (De: descartada).

Se analizaron 2262 lances de pesca en donde se listaron todas las especies de peces observadas entre el 2003 y 


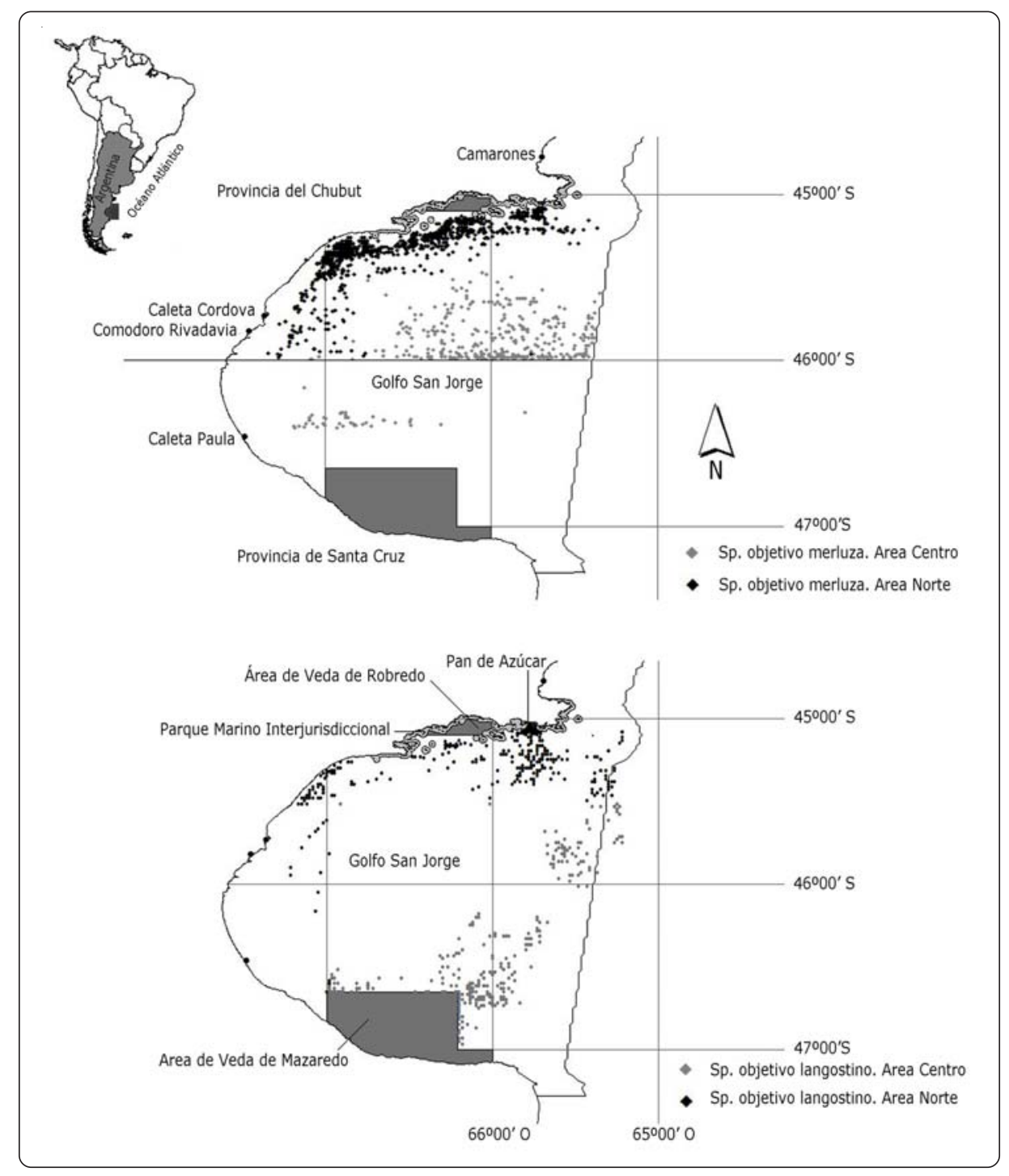

Figura 1. Lances analizados y caracterizados por especie objetivo y área de pesca. Flota fresquera de altura, Programa de Observadores a Bordo de la Provincia del Chubut, período 2003-2012 / Hauls analysed and characterized by target species and fishing area. Ice- trawlers fleet, On-board Observer Program of the Chubut Province, during 2003-2012 June period

2012. Para la nomenclatura científica se utilizó el catálogo en versión electrónica de Eschmeyer (2013). La Frecuencia de Ocurrencia (FO) para cada taxón se calculó por especie objetivo y por área, como criterio para caracterizar la captura incidental (Hall 1996, Hall et al. 2000):

$\mathrm{FO}_{\text {taxón/sp. objetivo/área }}=$ (lances donde el taxón fue identificado / lances totales) 100

Los rangos de frecuencia de ocurrencia por taxón fueron construidos utilizando métodos de remuestreo con reemplazo (Crawley 2007).
Para el análisis descriptivo de las especies se definieron 2 áreas, un área que se denominó norte, donde se agruparon los lances ubicados aproximadamente a $50 \mathrm{~km}$ de la costa, y un área centro, donde se agruparon los lances que se realizaron en el centro del golfo, por fuera de los $50 \mathrm{~km}$. Estas 2 zonas se diferencian por la operatoria de pesca tanto en la pesquería de langostino como en la pesquería de merluza.

Se calculó la frecuencia en que el taxón fue observado como raro, común, abundante o dominante: 
$\mathrm{F}_{\mathrm{Ra}}=$ (lances donde el taxón fue Raro / lances donde el taxón fue identificado) 100

$\mathrm{F}_{\mathrm{Co}}=$ (lances donde el taxón fue Común / lances donde el taxón fue identificado) 100

$\mathrm{F}_{\mathrm{Ab}}=$ (lances donde el taxón fue Abundante / lances donde el taxón fue identificado) 100

$\mathrm{F}_{\mathrm{Do}}=$ (lances donde el taxón fue Dominante / lances donde el taxón fue identificado) 100

En los lances en que el taxón fue identificado, se calculó el porcentaje en que fue Totalmente encajonada (En) o Parcialmente encajonada (Pe) y Descartada totalmente (De):

$\mathrm{F}_{\mathrm{En}}=$ (lances donde el taxón fue En / lances donde el taxón fue identificado) 100

$\mathrm{F}_{\mathrm{Pe}}=$ (lances donde el taxón fue Pe / lances donde el taxón fue identificado) 100

$\mathrm{F}_{\mathrm{De}}=$ (lances donde el taxón fue De / lances donde el taxón fue identificado) 100

Se analizaron las asociaciones de peces y su distribución espacial con relación a la especie objetivo a partir de los datos de presencia/ausencia. Los datos de cada taxón por lance fueron agrupados en rectángulos de 6' x 6' para todos los años incluidos en el análisis (2003-2012), en forma similar al utilizado por Góngora (2011) que incluye 10 rectángulos en un grado. Se calculó a partir de la matriz de frecuencias absolutas por rectángulo, una matriz de frecuencias relativas, es decir dividiendo cada frecuencia absoluta por la cantidad de lances realizados en el rectángulo. Fueron eliminados del análisis los rectángulos con menos de 5 lances. Para cada año se incluyeron sólo las especies observadas en al menos 10 registros (Stobutzki et al. 2003), considerando que la ocurrencia de especies raras sería un problema de azar más que un indicador de condiciones ecológicas (Gauch 1989, Legendre \& Legendre 1998).

Se consideró también en el análisis la especie objetivo, es decir, un rectángulo puede ingresar a la tabla 2 veces, una con especie objetivo merluza y otra con la especie objetivo langostino. De este modo, además de analizar las áreas se determinó si la biodiversidad presente en la captura incidental varió según el arte de pesca. Se utilizó un análisis de componentes principales, que permitió ordenar las áreas de acuerdo a las especies presentes en cada uno de los años y a la especie objetivo. El análisis factorial se utilizó como una herramienta de exploración de la tabla. Se analizó el agrupamiento de los sitios y la representación de las especies. En ambos casos se incluyeron en el análisis los individuos (sitios) y las variables (taxones) que contribuyeron al análisis factorial en un porcentaje mayor al 1\% (Escofier \& Pagès 1992). Los cálculos se realizaron utilizando la librería FactoMineR (Lê et al. 2008) del programa R v2.9.0 (R Development Core Team 2006).

\section{Resultados}

La flota fresquera que operó en el Golfo San Jorge entre los años 2003 y junio de 2012 alternó mareas dirigidas a langostino y a merluza. Se observó una estratificación espacial en la distribución de los lances de pesca realizados por esta flota. La flota alterna 2 áreas de pesca, el centro y norte del Golfo San Jorge, para ambas especies objetivo (Fig. 1).

Se identificaron un total de 90 taxones capturados, 57 peces y 33 invertebrados. Los peces óseos fueron los más numerosos con 34 taxones, y dentro de los condrictios las rayas con 14 especies identificadas en las capturas (Tabla 1). Dentro de los invertebrados el principal grupo estuvo representado por los crustáceos (17 taxones), seguida por los moluscos (8 taxones) (Tabla 2).

\section{Merluza. Área norte del Golfo San Jorge}

En la captura de la flota fresquera, cuando operó dirigida a merluza en el área norte del golfo, se registraron 79 taxones de los cuales 10 presentaron una frecuencia de ocurrencia (FO) superior al 40\%: Merluccius hubbsi, Lithodes santolla, Illex argentinus, Munida gregaria, Stromateus brasiliensis, Callorhinchus callorynchus, Pleoticus muelleri, Zearaja chilensis, Patagonotothen ramsayi y Genypterus blacodes (Tabla 3). La merluza fue la especie más abundante en las capturas, consignándose en el $93 \%$ de los lances como dominante y 4,6\% como abundante. Peisos petrunkevitchi, P. muelleri, M. gregaria y $L$. santolla fueron registrados como dominantes pero con una FO menor al 3,7\%. M. gregaria fue abundante en el $7,7 \%$ de los lances en que fue identificada y $P$. petrunkevitchi en un 3,7\%. En tanto que 17 taxones se consignaron como comunes (Co), siendo L. santolla, M. gregaria, Engraulis anchoita y Z. chilensis las especies más importantes (entre el 5 y 10\% de los lances en que fueron identificadas) (Tabla 4).

La flota aprovechó el 53\% de los taxones (41 taxones) de forma parcial o total en algunos de los lances registrados en las capturas, pero 11 taxones fueron aprovechados en un porcentaje superior al 60\%: M. hubbsi, 
Tabla 1. Lista taxonómica de especies de peces capturadas por la flota fresquera de altura en el Golfo San Jorge en el período 2003-2012 / Taxonomic list of fish species caught by the fleet in the San Jorge Gulf during 20032012 period

\begin{tabular}{|c|c|}
\hline Myxini & Ophidiformes \\
\hline Myxiniformes & Ophidiidae \\
\hline Myxinidae & Genypterus blacodes Schnaider, 1801 \\
\hline Myxine australis Jenyns, 1842 & Genypterus brasiliensis Regan, 1903 \\
\hline Chondrichthyes & Raneya brasiliensis Miranda Ribeiro, 1903 \\
\hline Elasmobranchii & Batrachoidiformes \\
\hline Hexanchiformes & Batrachoididae \\
\hline Hexanchidae & Triathalassothia argentina Berg, 1897 \\
\hline Notorhynchus cepediamus (Perón, 1807) & Atheriniformes \\
\hline Carcharhiniformes & Atherinidae \\
\hline Scyliorhinidae & Odontesthes smitti Lahille, 1929 \\
\hline Schroederichthys bivius Müller y Henle, 1841 & Scorpaeniformes \\
\hline Triakidae & Sebastidae \\
\hline Mustelus schmitti Springer, 1939 & Sebastes oculatus Cuvier, 1833 \\
\hline Gaeleorhimus galeus Linné, 1758 & Congiopodidae \\
\hline Squaliformes & Congiopodus peruviamus (Cuvier, 1829) \\
\hline Squalidae & Triglidae \\
\hline Squalus acanthias Linné, 1758 & Prionotus nudigula Ginsburg, 1950 \\
\hline Squalus mitsukurii Jordan y Snyder, 1903 & Agonidae \\
\hline Squatiniformes & Agonopsis chiloensis (Jenyns, 1842) \\
\hline Squatinidae & Perciformes \\
\hline Squatina guggenheim (Marini, 1930) & Polyprionidae \\
\hline Torpediniformes & Polyprion americamus Bloch y Schneider, 1801 \\
\hline Narcinidae & Serranidae \\
\hline Discopyge tschudii Heckel, 1846 & Acanthistius patachonicus (Jenyns, 1842) \\
\hline Torpedo puelcha Lahille, 1926 & Carangidae \\
\hline Rajiformes & Parona signata (Jenyns, 1842) \\
\hline Rajidae & Bramidae \\
\hline Atlantoraja cyclophora Regan, 1903 & Brama brama (Bonnaterre, 1788) \\
\hline Atlantoraja castelnaui Miranda Ribeiro, 1907 & Sciaenidae \\
\hline Dipturus trachyderma (Krefft y Stehmann, 1975) & Cynoscion guatucupa (Cuvier, 1829) \\
\hline Dipturus argentinesis Diaz de Astarloa, Mabragaña, Hanner y Figueroa, 2008 & Cheilodactylidae \\
\hline Bathyraja brachyurops (Fowler, 1910) & Nemadactylus bergi (Norman, 1937) \\
\hline Bathyraja macloviana (Norman, 1913) & Mullidae \\
\hline Psammobatis normani McEachran, 1983 & Mullus argentinae Hubbs \& Marini, 1933 \\
\hline Psammobatis lentiginosa McEachran, 1983 & Zoarcidae \\
\hline Psammobatis rudis Gunther, 1870 & Austrolycus laticinctus (Berg, 1895) \\
\hline Sympterygia bonapartii Muller y Henle, 1841 & Bovichthidae \\
\hline Zearaja chilensis (Guichenot, 1848) & Cottoperca trigloides (Günther, 1881) \\
\hline Myliobatiformes & Nototheniidae \\
\hline Myliobatidae & Patagonotothen ramsayi (Regan, 1913) \\
\hline Myliobatis goodei Garman, 1885 & Eleginopsidae \\
\hline Holocephalii & Eleginops maclovinus (Valenciennes, 1830) \\
\hline Chimaeriformes & Percophidae \\
\hline Callorhynchidae & Percophis brasiliensis (Quoy y Gaimard, 1824) \\
\hline Callorhinchus callorynchus Linné 1758 & Pinguipedidae \\
\hline Osteichthyes & Pinguipes brasiliamus Cuvier 1829 \\
\hline Clupeiformes & Scombridae \\
\hline Engraulidae & Pseudopercis semifasciata (Cuvier, 1829) \\
\hline Engraulis anchoita Hubbs y Marini, 1935 & Centrolophidae \\
\hline Gadiformes & Seriolella porosa Guichenot, 1848 \\
\hline Moridae & Stromateidae \\
\hline Salilota australis Günther, 1878 & Stromateus brasiliensis Fowler, 1906 \\
\hline Phycidae & Pleuronectiformes \\
\hline Urophycis brasiliensis Kaup, 1858 & Paralichthyidae \\
\hline Merlucciidae & Xystreurys rasile (Jordan, 1890) \\
\hline Merluccius hubbsi Marini, 1933 & Paralichthys patagonicus Jordan, 1889 \\
\hline Macruronidae & Paralichthys isosceles Jordan, 1891 \\
\hline Macruromus novaezelandiae Lonnberg, 1907 & \\
\hline
\end{tabular}


Tabla 2. Lista taxonómica de invertebrados capturados por la flota fresquera de altura en el Golfo San Jorge en el período 2003-2012 / Taxonomic list of invertebrates captured by the fleet in San Jorge Gulf during 20032012 period

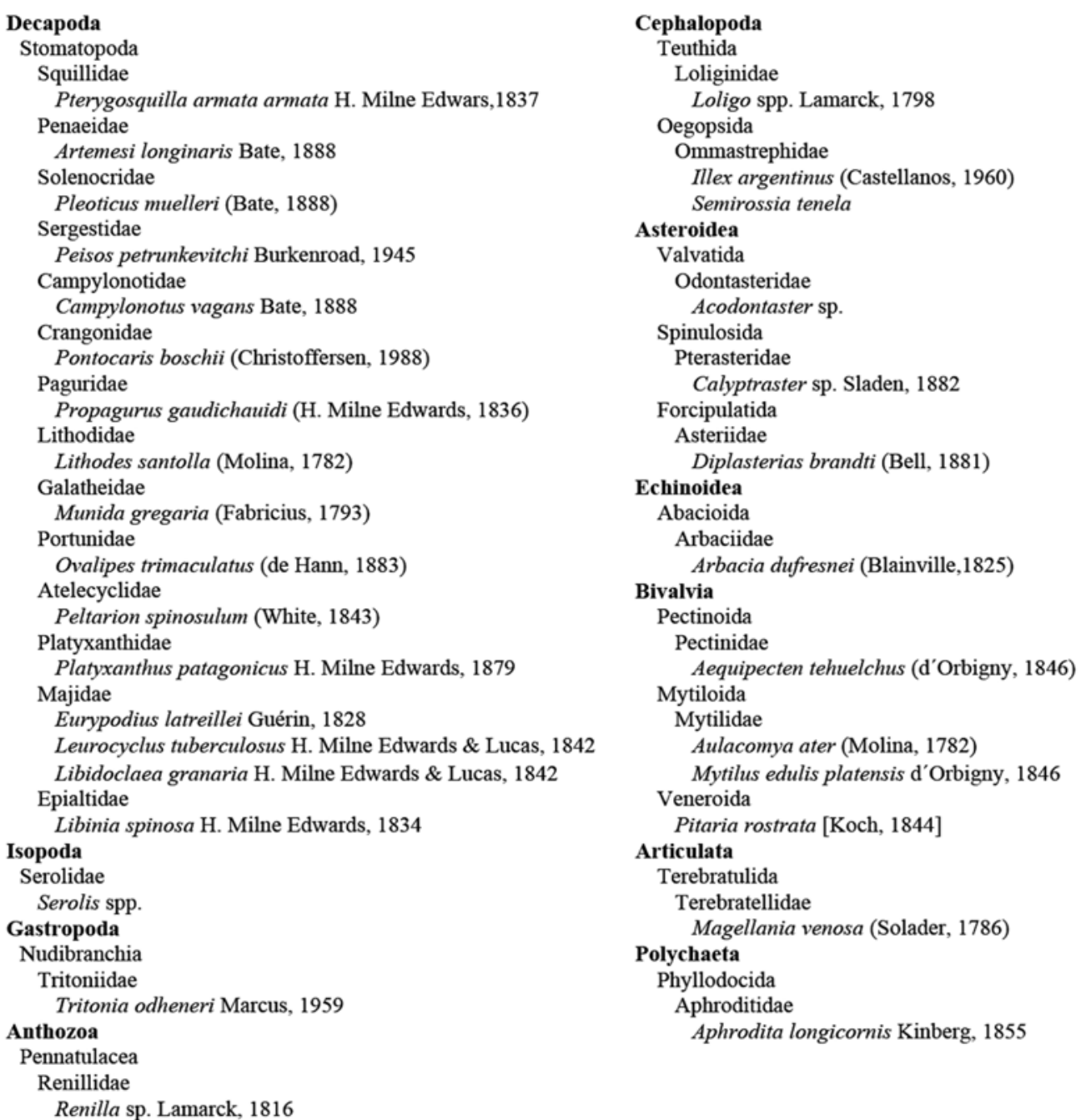

L. santolla, C. callorynchus, P. muelleri, Z. chilensis, S. bonapartii, Dipturus trachyderma, Eleginops maclovinus, Notorhynchus cepedianus, Scomber japonicus y Macruronus novaezelandiae (Tabla 5).

\section{Merluza. Área centro del Golfo San Jorge}

Se registraron menos taxones en las capturas del centro del golfo en comparación a las del norte con un total de 60 taxones, de los cuales 14 presentaron una FO superior al 40\%: M. hubbsi, Z. chilensis, P. ramsayi, S. bonapartii, C. callorynchus, Psammobatis normani, Squalus acanthias, Congiopodus peruvianus, S. brasiliensis, G. blacodes, Xystreurys rasile, I. argentinus, Loligo spp. y
L. santolla (Tabla 3). En cuanto a la abundancia sólo la especie objetivo fue consignada como dominante, mientras que sólo se registraron abundanteen forma esporádica C. callorynchus y $P$. ramsayi. Comunes en las capturas se registraron 15 taxones de los cuales $C$. callorynchus, $Z$. chilensis y Munida gregaria presentaron la mayor frecuencia (Tabla 4). El 60\% de los taxones registrados en las capturas fueron aprovechados parcial o totalmente en algún lance, pero sólo 11 taxones fueron con un porcentaje mayor al 60\%: M. hubbsi, Z. chilensis, I. argentinus, S. bonapartii, C. callorynchus, D. trachyderma, Parona signata, Eleginops maclovinus, Paralichthys isosceles, Atlantoraja castelnaui, Galeorhinus galeus, Brama brama y P. muelleri (Tabla 5). 


\section{LANGostino. ÁreA NORTE dEL Golfo SAN JoRge}

En las capturas de la flota fresquera cuando operó dirigida a langostino en el norte del golfo se registraron 71 taxones, de los cuales 11 presentaron una FO superior al 40\%: $M$. hubbsi, P. muelleri, L. santolla, M. gregaria, Loligo spp., Renilla sp., S. brasiliensis, P. normani, $P$. ramsayi, $I$. argentinus y G. blacodes (Tabla 3). Desde el punto de vista de la abundancia $P$. muelleri fue consignada como dominante en el 40,4\% de los lances en que fue observada, siguiéndo la merluza (24,9\%) y M. gregaria (7,8\%). Como abundante fueron consignados 5 taxones, de los cuales la merluza (28,4\%) y el langostino (26,8\%) fueron los más importantes, mientras que 11 taxones fueron consignados como comunes, siendo M. gregaria, Renilla sp., $P$. muelleri, Peisos petrunkevitchi y la merluza los más importantes (Tabla 4). En relación al aprovechamiento de la captura, cuando la flota operó dirigida a langostino, sólo el 19\% de los taxones fueron aprovechados parcial o totalmente, siendo los más importantes $P$. muelleri y $L$. santolla con porcentajes mayores al $40 \%$ de los lances en los que fueron registrados. Por otro lado, Genypterus brasiliensis y Polyprion americanus fueron aprovechados total o parcialmente en un porcentaje mayor o igual al 50\%, pero su FO fue muy baja (Tabla 5).

\section{LANGostino. Área CENTRO del Golfo SAN JoRgE}

En las capturas se registraron 50 taxones, de los cuales únicamente 10 presentaron una FO mayor al 40\%: $M$. hubbsi, P. muelleri, G. blacodes, P. ramsayi, Z. chilensis, Pterygosquilla armata armata, Loligo spp., L. santolla,

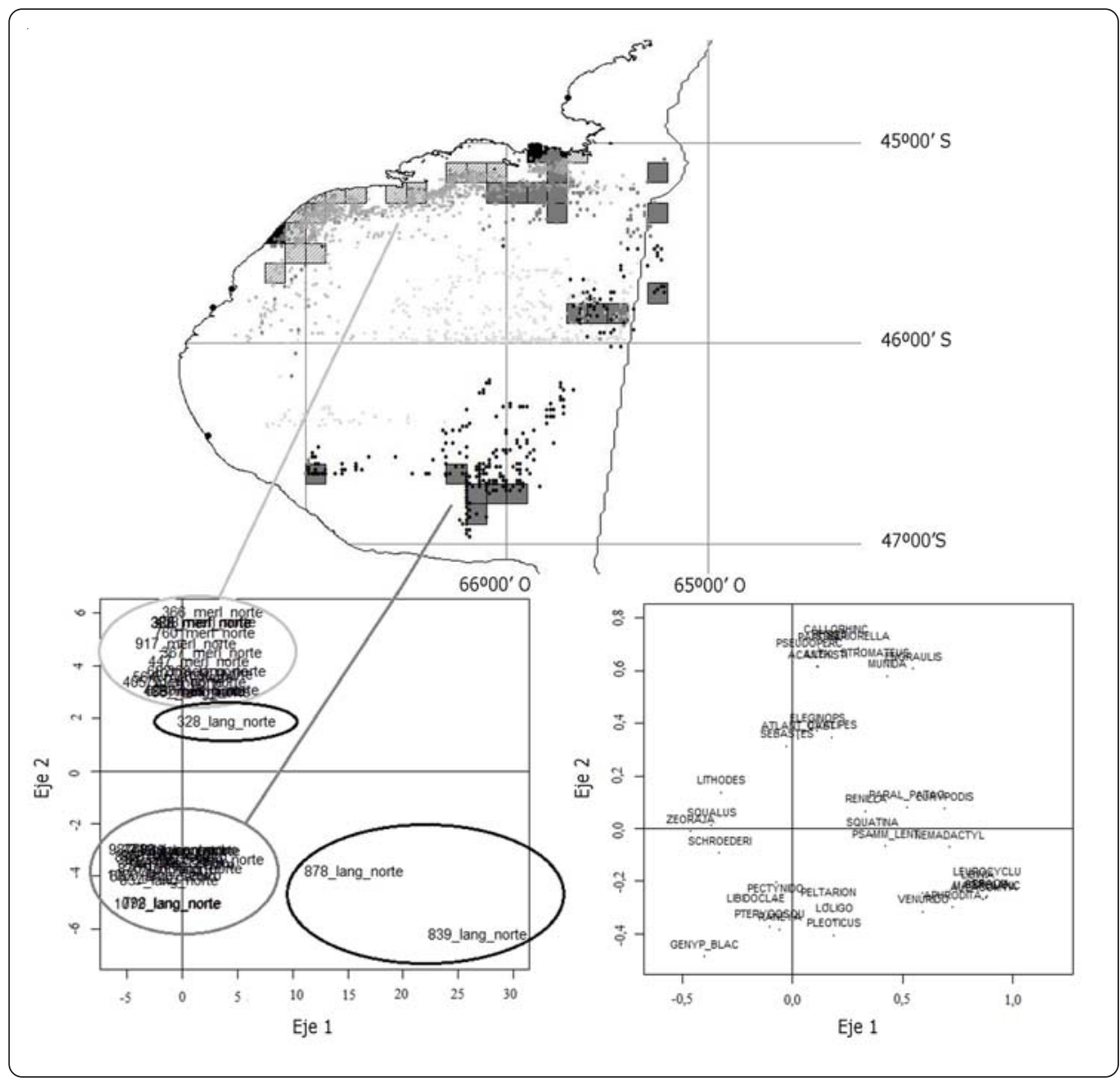

Figura 2. Análisis de componentes principales: áreas (individuos) que contribuyen a los 2 principales factores y representación de las variables. Para mejorar la visualización se presentan sólo los puntos, se eliminaron las líneas que indicaban los vectores y el círculo de correlación / Principal component analysis: areas (individuals) that contribute to the 2 main factors and variables representing. To improve visualization only points are showed, lines were removed indicating vectors and the correlation circle 
Tabla 3. Frecuencia de ocurrencia (FO) por especie, área y especie objetivo. n: número de lances en los que la especie fue identificada, \%: FO en los lances de pesca, 0,05 y 0,95: intervalos de confianza al 0,05 y 0,95 / Frequency of occurrence (FO) by species, area and target species. $\mathrm{n}$ : number of hauls in which the species was identified, \%: FO in fishing hauls, 0.05 and 0.95 : confidence intervals 0.05 to 0.95

\begin{tabular}{|c|c|c|c|c|c|c|c|c|c|c|c|c|c|c|c|c|}
\hline & \multirow{2}{*}{\multicolumn{4}{|c|}{$\begin{array}{l}\text { Langostino, Área centro } \\
\mathrm{N}^{\circ} \text { de lances }=364\end{array}$}} & \multirow{2}{*}{\multicolumn{4}{|c|}{$\begin{array}{c}\text { Langostino, Área norte } \\
\mathrm{N}^{\circ} \text { de lances }=401\end{array}$}} & \multirow{2}{*}{\multicolumn{4}{|c|}{$\begin{array}{c}\text { Merluza, Área centro } \\
\mathrm{N}^{\circ} \text { de lances }=381\end{array}$}} & \multirow{2}{*}{\multicolumn{4}{|c|}{$\begin{array}{l}\text { Merluza, Área norte } \\
\mathrm{N}^{\circ} \text { de lances }=1116\end{array}$}} \\
\hline & & & & & & & & & & & & & & & & \\
\hline & $\mathrm{n}$ & $\%$ & 0,05 & 0,95 & $\mathrm{n}$ & $\%$ & 0,05 & 0,95 & $\mathrm{n}$ & $\%$ & 0,05 & 0,95 & $\mathrm{n}$ & $\%$ & 0,05 & 0,95 \\
\hline Merluccius hubbsi & 363 & 99,7 & 99,18 & 100 & 377 & 94,0 & 92,52 & 95,26 & 374 & 98,2 & 97,38 & 98,95 & 1095 & 98,1 & 97,76 & 98,39 \\
\hline Pleoticus muelleri & 353 & 97,0 & 95,33 & 98,35 & 357 & 89,0 & 86,78 & 91,27 & 140 & 36,7 & 32,55 & 40,68 & 674 & 60,4 & 58,60 & 63,26 \\
\hline Lithodes santolla & 353 & 97,0 & 95,33 & 98,35 & 300 & 74,8 & 71,32 & 78,05 & 349 & 91,6 & 89,50 & 93,70 & 1049 & 94,0 & 93,01 & 94,98 \\
\hline Genypterus blacodes & 310 & 85,2 & 82,14 & 88,19 & 162 & 40,4 & 36,41 & 44,39 & 201 & 52,8 & 48,56 & 56,96 & 470 & 42,1 & 39,52 & 44,35 \\
\hline Zearaja chilensis & 222 & 61,0 & 56,87 & 65,11 & 148 & 36,9 & 32,92 & 40,65 & 332 & 87,1 & 84,78 & 90,03 & 672 & 60,2 & 57,80 & 62,55 \\
\hline Patagonotothen ramsayi & 199 & 54,7 & 50,27 & 58,79 & 179 & 44,6 & 40,65 & 48,63 & 260 & 68,2 & 64,30 & 72,18 & 628 & 56,3 & 53,85 & 58,69 \\
\hline Pterygosquilla armata armata & 188 & 51,6 & 47,25 & 56,04 & 45 & 11,2 & 8,73 & 13,97 & 6 & 1,6 & 0,52 & 2,62 & 146 & 2,3 & 11,47 & 14,78 \\
\hline Loligo spp. & 187 & 51,4 & 46,98 & 55,77 & 218 & 54,4 & 50,37 & 58,35 & 170 & 44,6 & 40,42 & 48,82 & 311 & 27,9 & 25,72 & 30,11 \\
\hline Libidoclaea granaria & 160 & 44,0 & 39,84 & 48,35 & 155 & 38,7 & 34,66 & 42,64 & 141 & 37,0 & 33,07 & 40,94 & 201 & 18,0 & 16,13 & 19,89 \\
\hline Illex argentimus & 153 & 42,0 & 37,91 & 46,43 & 163 & 40,6 & 36,66 & 44,64 & 262 & 68,8 & 64,83 & 72,70 & 922 & 82,6 & 80,73 & 84,41 \\
\hline Sympterygia bonapartii & 144 & 39,6 & 35,44 & 43,96 & 79 & 19,7 & 16,46 & 22,94 & 256 & 67,2 & 62,99 & 70,87 & 246 & 22,0 & 19,98 & 24,10 \\
\hline Psammobatis normani & 115 & 31,6 & 27,47 & 35,71 & 181 & 45,1 & 41,15 & 49,13 & 236 & 61,9 & 58,01 & 66,14 & 442 & 39,6 & 37,19 & 42,03 \\
\hline Stromateus brasiliensis & 111 & 30,5 & 26,65 & 34,62 & 204 & 50,9 & 46,88 & 54,86 & 204 & 53,5 & 49,34 & 57,74 & 716 & 64,2 & 61,83 & 66,49 \\
\hline Peltarion spinosulum & 95 & 26,1 & 22,25 & 29,95 & 156 & 38,9 & 34,91 & 42,89 & 24 & 6,3 & 3,41 & 7,09 & 178 & 15,9 & 14,16 & 17,83 \\
\hline Xystreurys rasile & 77 & 21,2 & 17,58 & 24,73 & 117 & 29,2 & 25,44 & 32,92 & 174 & 45,7 & 41,73 & 50,13 & 343 & 30,7 & 28,49 & 32,97 \\
\hline Discopyge tschudii & 61 & 16,8 & 13,74 & 20,05 & 105 & 26,2 & 22,69 & 29,93 & 97 & 25,5 & 21,78 & 29,13 & 128 & 11,5 & 9,95 & 13,08 \\
\hline Renilla sp. & 48 & 13,2 & 10,43 & 16,21 & 209 & 52,1 & 48,13 & 56,11 & 83 & 21,8 & 18,37 & 25,20 & 338 & 30,3 & 28,05 & 32,53 \\
\hline Munida gregaria & 45 & 12,4 & 9,62 & 15,11 & 219 & 54,6 & 50,62 & 58,60 & 33 & 8,7 & 6,30 & 11,02 & 796 & 71,3 & 69,18 & 73,48 \\
\hline Callorhinchus callorynchus & 44 & 12,1 & 9,34 & 14,84 & 145 & 36,2 & 32,42 & 40,15 & 238 & 62,5 & 58,27 & 66,40 & 692 & 62,0 & 59,68 & 64,34 \\
\hline Galeorhimus galeus & 31 & 8,5 & 6,32 & 10,99 & 18 & 4,5 & 2,74 & 6,23 & 79 & 20,7 & 17,32 & 24,15 & 75 & 6,7 & 5,56 & 7,97 \\
\hline Mustelus schmitti & 27 & 7,4 & 5,22 & 9,62 & 2 & 0,5 & 0,00 & 1,25 & 38 & 10,0 & 7,61 & 12,60 & 86 & 7,7 & 6,45 & 9,05 \\
\hline Percophis brasiliensis & 27 & 7,4 & 5,22 & 9,62 & & & & & & & & & 10 & 0,9 & 0,45 & 1,34 \\
\hline Paralichthys isosceles & 26 & 7,1 & 4,95 & 9,34 & 65 & 16,2 & 13,22 & 19,20 & 24 & 6,3 & 4,46 & 8,40 & 101 & 9,1 & 7,71 & 10,48 \\
\hline Acanthistius patachonicus & 26 & 7,1 & 4,95 & 9,34 & 16 & 4,0 & 2,49 & 5,74 & 25 & 6,6 & 4,46 & 8,66 & 380 & 34,1 & 31,72 & 36,38 \\
\hline Schroederichthys bivius & 25 & 6,9 & 4,67 & 9,07 & 19 & 4,7 & 2,99 & 6,48 & 95 & 24,9 & 21,26 & 28,61 & 73 & 6,5 & 5,29 & 7,80 \\
\hline Nemadactylus bergi & 24 & 6,6 & 4,40 & 8,79 & 136 & 33,9 & 29,93 & 37,66 & 5 & 1,3 & 0,52 & 2,36 & 25 & 2,2 & 1,52 & 2,96 \\
\hline Parona signata & 24 & 6,6 & 4,40 & 8,79 & 16 & 4,0 & 2,49 & 5,74 & 51 & 13,4 & 10,50 & 16,27 & 291 & 26,1 & 24,01 & 28,23 \\
\hline Congiopodus peruvianus & 22 & 6,0 & 4,12 & 8,24 & 27 & 6,7 & 4,74 & 8,73 & 210 & 55,1 & 50,92 & 59,06 & 85 & 7,6 & 6,36 & 8,96 \\
\hline Platyxhantus patagonicus & 19 & 5,2 & 3,30 & 7,14 & 31 & 7,7 & 5,74 & 9,98 & 43 & 11,3 & 8,66 & 13,92 & 143 & 12,8 & 11,20 & 14,43 \\
\hline Squalus acanthias & 19 & 5,2 & 3,30 & 7,14 & 22 & 5,5 & 3,74 & 7,48 & 232 & 60,9 & 56,96 & 64,83 & 112 & 10,0 & 8,60 & 11,56 \\
\hline Raneya brasiliensis & 18 & 4,9 & 3,02 & 6,87 & 37 & 9,2 & 6,98 & 11,72 & & & & & 4 & 0,4 & 0,09 & 0,72 \\
\hline Myxine australis & 13 & 3,6 & 1,92 & 5,2 & 10 & 2,5 & 1,25 & 3,74 & 3 & 0,8 & 0,00 & 1,57 & 1 & 0,1 & 0,00 & 0,27 \\
\hline Peisos petrunkevitchi & 12 & 3,3 & 1,92 & & 22 & 5,5 & 3,74 & 7,48 & 5 & 1,3 & 0,52 & & 272 & 24,4 & 22,22 & 26,52 \\
\hline Eleginops maclovinus & 11 & 3,0 & 1,65 & 4,67 & 22 & 5,5 & 3,74 & 7,48 & 25 & 6,6 & 4,46 & 8,66 & 103 & 9,2 & 7,89 & 10,66 \\
\hline Psammobatis rudis & 10 & 2,7 & 1,37 & 4, & 12 & 3,0 & 1,75 & 4,49 & 6 & 1,6 & 0,52 & 2,62 & 23 & 2,1 & 1,34 & 2,78 \\
\hline Seriolella porosa & 8 & 2,2 & 1,10 & 3,57 & 30 & 7,5 & 5,24 & 9,73 & 22 & 5,8 & 3,94 & 7,87 & 299 & 26,8 & 24,64 & 28,94 \\
\hline Sebastes oculatus & 8 & 2,2 & 1,10 & 3,57 & 19 & 4,7 & 2,99 & 6,48 & 22 & 5,8 & 3,94 & 7,87 & 83 & 7,4 & 6,18 & 8,69 \\
\hline Odontesthes smitti & 5 & 1,4 & 0,55 & 2,47 & 24 & 6,0 & 3,99 & 7,98 & & & & & 6 & 0,5 & 0,18 & 0,90 \\
\hline Pseudopercis semifasciata & 5 & 1,4 & 0,55 & 2,47 & 13 & 3,2 & 1,75 & 4,74 & 30 & 7,9 & 5,77 & 10,24 & 180 & 16,1 & 14,34 & 17,92 \\
\hline Cottoperca trigloides & 5 & 1,4 & 0,55 & 2,47 & 9 & 2,2 & 1,00 & 3,49 & 90 & 23,6 & 20,21 & 27,30 & 48 & 4,3 & 3,32 & 5,29 \\
\hline Pinguipes brasiliamus & 5 & 1,4 & 0,27 & 2,20 & 7 & 1,7 & 0,75 & 2,99 & & & & & 41 & 3,7 & 2,78 & 4,57 \\
\hline Salilota australis & 5 & 1,4 & 0,55 & 2,47 & 5 & 1,2 & 0,50 & 2,24 & 100 & 26,2 & 22,57 & 29,92 & 73 & 6,5 & 5,38 & 7,80 \\
\hline Bathyraja macloviana & 4 & 1,1 & 0,27 & 1,92 & 14 & 3,5 & 2,00 & 4,99 & 31 & 8,1 & 6,02 & 10,50 & 37 & 3,3 & 2,42 & 4,21 \\
\hline Engraulis anchoita & 1 & 0,3 & 0,00 & 0,82 & 42 & 10,5 & 7,98 & 12,97 & 2 & 0,5 & 0,00 & 1,31 & 228 & 20,4 & 18,73 & 22,67 \\
\hline Tritonia adheneri & 1 & 0,3 & 0,00 & 0,82 & 19 & 4,7 & 2,99 & 6,48 & 27 & 7,1 & 4,99 & 9,45 & 36 & 3,2 & 2,42 & 4,12 \\
\hline
\end{tabular}

Libidoclaea granaria e I. argentinus (Tabla 3). Sólo 4 taxones fueron identificados como dominantes y 6 como abundantes, siendo M. hubbsi y P. muelleri los más importantes (Tabla 4). Se identificaron 8 taxones comunes, de los cuales M. hubbsi, P. muelleri y L. santolla, nuevamente fueron los más importantes. Únicamente 10 taxones (19\%) fueron aprovechados parcial o totalmente, siendo los más importantes P. muelleri y L. santolla con un aprovechamiento superior al $60 \%$, mientras que $M$. hubbsi y Xystreurys rasile sólo se aprovecharon en el $30 \%$ de los lances en los que fueron consignados (Tabla 5). 
Continuación Tabla 3 / Table 3 Continued

\begin{tabular}{|c|c|c|c|c|c|c|c|c|c|c|c|c|c|c|c|c|}
\hline & \multirow{2}{*}{\multicolumn{4}{|c|}{$\begin{array}{c}\text { Langostino, Área centro } \\
\mathrm{N}^{\circ} \text { de lances }=364\end{array}$}} & \multirow{2}{*}{\multicolumn{4}{|c|}{$\begin{array}{c}\text { Langostino, Área norte } \\
\mathrm{N}^{\circ} \text { de lances }=401\end{array}$}} & \multirow{2}{*}{\multicolumn{4}{|c|}{$\begin{array}{c}\text { Merluza, Área centro } \\
\mathrm{N}^{\circ} \text { de lances }=381\end{array}$}} & \multirow{2}{*}{\multicolumn{4}{|c|}{$\begin{array}{l}\text { Merluza, Área norte } \\
\mathrm{N}^{\circ} \text { de lances }=1116\end{array}$}} \\
\hline & & & & & & & & & & & & & & & & \\
\hline & $\mathrm{n}$ & $\%$ & 0,05 & 0,95 & $\mathrm{n}$ & $\%$ & 0,05 & 0,95 & $\mathrm{n}$ & $\%$ & 0,05 & 0,95 & $\mathrm{n}$ & $\%$ & 0,05 & 0,95 \\
\hline Agonopsis chiloensis & 1 & 0,3 & 0,00 & 0,82 & 9 & 2,2 & 2,49 & 5,74 & & & & & 7 & 0,6 & 0,27 & 1,08 \\
\hline Myliobatis goodei & 1 & 0,3 & 0,00 & 0,82 & 6 & 1,5 & 0,50 & 2,49 & 2 & 0,5 & 0,00 & 1,31 & 12 & 1,1 & 0,63 & 1,61 \\
\hline Genypterus brasiliensis & 1 & 0,3 & 0,00 & 0,82 & 4 & 1,0 & 0,25 & 1,75 & 11 & 2,9 & 1,57 & 4,46 & 30 & 2,7 & 1,88 & 3,41 \\
\hline Brama brama & 1 & 0,3 & 0,00 & 0,82 & & & & & 28 & 7,3 & 5,25 & 9,71 & 11 & 1,0 & 0,54 & 1,52 \\
\hline Campylonotus vagans & 1 & 0,3 & 0,00 & 0,82 & & & & & & & & & 7 & 0,6 & 0,27 & 0,99 \\
\hline Aphrodita longicornis & & & & & 125 & 31,2 & 27,43 & 34,91 & 3 & 0,8 & 0,26 & 1,57 & 10 & 0,9 & 0,45 & 1,34 \\
\hline Leurocyclus tuberculosus & & & & & 99 & 24,7 & 21,20 & 28,18 & 1 & 0,3 & 0,00 & 0,79 & 9 & 0,8 & 0,36 & 1,25 \\
\hline Petaria rostrata & & & & & 40 & 10,0 & & & & & & & & & & \\
\hline Magallanica venosa & & & & & 35 & 8,7 & 6,48 & 11,22 & & & & & & & & \\
\hline Arbacia dufresnei & & & & & 34 & 8,5 & 6,23 & 10,97 & & & & & & & & \\
\hline Aulacomya ater & & & & & 29 & 7,2 & 4,99 & 9,48 & & & & & & & & \\
\hline Serolis sp. & & & & & 20 & 5,0 & 3,24 & 6,73 & & & & & & & & \\
\hline Euripodis latreillei & & & & & 15 & 3,7 & 2,24 & 5,49 & & & & & 25 & 2,2 & 1,52 & 2,96 \\
\hline Psammobatis lentiginosa & & & & & 12 & 3,0 & 1,75 & 4,49 & 18 & 4,7 & 3,15 & 6,56 & 16 & 1,4 & 0,90 & 2,06 \\
\hline Libinia spinosa & & & & & 10 & 2,5 & 1,25 & 3,74 & & & & & 1 & 0,1 & 0,00 & 0,27 \\
\hline Squatina guggenheim & & & & & 9 & 2,2 & 1,00 & 3,49 & 4 & 1,0 & 0,26 & 2,10 & 13 & 1,2 & 0,63 & 1,70 \\
\hline Acodontaster $\mathrm{sp}$. & & & & & 9 & 2,2 & 1,00 & 3,49 & & & & & & & & \\
\hline Paralichthys patagonicus & & & & & 8 & 2,0 & 1,00 & 3,24 & & & & & 28 & 2,5 & 1,79 & 3,32 \\
\hline Macruromus novaezelandiae & & & & & 8 & 2,0 & 1,00 & 3,24 & 14 & 3,7 & 2,10 & 5,25 & 3 & 0,3 & 0,00 & 0,54 \\
\hline Semirossia tenela & & & & & 7 & 1,7 & 0,75 & 2,99 & & & & & & & & \\
\hline Ovalipes trimaculatus & & & & & 5 & 1,2 & 0,50 & 2,24 & & & & & 7 & 0,6 & 0,27 & 1,08 \\
\hline Triathalassothia argentina & & & & & 4 & 1,0 & 0,25 & 1,75 & 1 & 0,3 & 0,00 & 0,79 & 1 & 0,1 & 0,00 & 0,27 \\
\hline Aequipecten tehuelchus & & & & & 3 & 0,7 & 0,25 & 1,50 & & & & & & & & \\
\hline Dipturus trachyderma & & & & & 1 & 0,2 & 0,00 & 0,75 & 94 & 24,7 & 21,00 & 28,35 & 115 & 10,3 & 8,78 & 11,83 \\
\hline Polyprion americamus & & & & & 1 & 0,2 & 0,00 & 0,75 & 2 & 0,5 & 0,00 & 1,31 & 12 & 1,1 & 0,63 & 1,61 \\
\hline Cynoscion guatucupa & & & & & 1 & 0,2 & 0,00 & 0,75 & & & & & 2 & 0,2 & 0,00 & 0,45 \\
\hline Mullus argentinae & & & & & 1 & 0,2 & 0,00 & 0,75 & & & & & 2 & 0,2 & 0,00 & 0,45 \\
\hline Calyptraster sp. & & & & & 1 & 0,2 & 0,00 & 0,75 & & & & & & & & \\
\hline Mytilus edulis platensis & & & & & 1 & 0,2 & 0,00 & 0,75 & & & & & & & & \\
\hline Atlantoraja castelnaui & & & & & & & & & 12 & 3,1 & 1,84 & 4,72 & 35 & 3,1 & 2,33 & 4,03 \\
\hline Bathyraja brachyurops & & & & & & & & & 9 & 2,4 & 1,05 & 3,67 & 22 & 2,0 & 1,34 & 2,69 \\
\hline Atlantoraja cyclophora & & & & & & & & & 16 & 4,2 & 2,62 & 6,04 & 21 & 1,9 & 1,25 & 2,60 \\
\hline Prionotus nudigula & & & & & & & & & & & & & 12 & 1,1 & 0,63 & 1,61 \\
\hline Dipturus argentinensis & & & & & & & & & 3 & 0,8 & 0,26 & 1,57 & 10 & 0,9 & 0,45 & 1,34 \\
\hline Notorhynchus cepedianus & & & & & & & & & 1 & 0,3 & 0,00 & 0,79 & 5 & 0,4 & 0,18 & 0,81 \\
\hline Propagurus gaudichauidi & & & & & & & & & & & & & 3 & 0,3 & 0,09 & 0,54 \\
\hline Diplasterias brandti & & & & & & & & & & & & & 2 & 0,2 & 0,00 & 0,45 \\
\hline Urophycis brasiliensis & & & & & & & & & & & & & 2 & 0,2 & 0,00 & 0,45 \\
\hline Squalus mitsukurii & & & & & & & & & 6 & 1,6 & 0,52 & 2,62 & 1 & 0,1 & 0,00 & 0,27 \\
\hline Artemesia longinaris & & & & & & & & & & & & & 1 & 0,1 & 0,00 & 0,27 \\
\hline Pontocaris boschii & & & & & & & & & & & & & 1 & 0,1 & 0,00 & 0,27 \\
\hline Scomber japonicus & & & & & & & & & & & & & 1 & 0,1 & 0,00 & 0,27 \\
\hline Torpedo puelcha & & & & & & & & & & & & & 1 & 0,1 & 0,00 & 0,27 \\
\hline Trypilaster sp. & & & & & & & & & & & & & 1 & 0,1 & 0,00 & 0,27 \\
\hline Austrolycus laticinctus & & & & & & & & & 1 & 0,3 & 0,00 & 0,79 & & & & \\
\hline
\end{tabular}

\section{ANÁlisis MUlTivariado de LA ASOCIACIÓN DE TAXONES Y ÁREAS}

La matriz de frecuencias relativas para analizar la asociación de peces y áreas fue de 105 rectángulos o sitios (individuos) por 71 especies (variables). Diecinueve sitios fueron visitados por buques que operaron sobre merluza o langostino, estos sitios fueron incorporados 2 veces caracterizados por la especie objetivo. La tabla resultante contó con 124 individuos (especie objetivositio). La matriz rectángulo-especie, tiene 8.946 celdas, de las cuales 5.396 (60\%) son valores cero, es decir la especie no fue registrada en ese rectángulo.

El porcentaje de inercia explicado por el primer eje fue de 13,9\% y con los 4 primeros ascendió a 37,8\% (Tabla 6). Según Escofier \& Pagès (1992) los porcentajes deben ser juzgados en función del tamaño de la tabla, por ejemplo 
Tabla 4. Frecuencia de ocurrencia por categoría de abundancia, área y especie objetivo: dominante (Do), abundante (Ab), común (Co) y rara (Ra) / Frequency of occurrence for each category of abundance, area and target species: dominant (Do), abundant (Ab), common (Co) and rare ( $\mathrm{Ra})$

\begin{tabular}{|c|c|c|c|c|c|c|c|c|c|c|c|c|c|c|c|c|}
\hline & \multicolumn{8}{|c|}{ Langostino } & \multicolumn{8}{|c|}{ Merluza } \\
\hline & \multicolumn{4}{|c|}{ Área centro } & \multicolumn{4}{|c|}{ Área norte } & \multicolumn{4}{|c|}{ Área centro } & \multicolumn{4}{|c|}{ Área norte } \\
\hline & Do & $\mathrm{Ab}$ & Co & $\mathrm{Ra}$ & Do & $\mathrm{Ab}$ & Co & $\mathrm{Ra}$ & Do & $\mathrm{Ab}$ & Co & $\mathrm{Ra}$ & Do & $\mathrm{Ab}$ & Co & $\mathrm{Ra}$ \\
\hline Acanthistius patachonicus & & & & 100 & & & & 100 & & & & 100 & & & 0,5 & 99 \\
\hline Acodontaster sp. & & & & & & & & 100 & & & & & & & & \\
\hline Aequipecten tehuelchus & & & & & & & & 100 & & & & & & & & \\
\hline Agonopsis chiloensis & & & & 100 & & & & 100 & & & & & & & & 100 \\
\hline Pitaria rostrata & & & & & & & & 100 & & & & & & & & \\
\hline Aphrodita longicornis & & & & & & & 0,8 & 99,2 & & & & 100 & & & & 100 \\
\hline Arbacia dufresnei & & & & & & & & 100 & & & & & & & & \\
\hline Artemesia longinaris & & & & & & & & & & & & & & & & 100 \\
\hline Atlantoraja castelnaui & & & & & & & & & & & & 100 & & & & 100 \\
\hline Atlantoraja cyclophora & & & & & & & & & & & & 100 & & & & 100 \\
\hline Aulacomya ater & & & & & & & & 100 & & & & & & & & \\
\hline Austrolycus laticinctus & & & & & & & & & & & & 100 & & & & \\
\hline Bathyraja brachyurops & & & & & & & & & & & & 100 & & & & 100 \\
\hline Bathyraja macloviana & & & & 100 & & & & 100 & & & & 100 & & & & 100 \\
\hline Brama brama & & & & 100 & & & & & & & & 100 & & & & 100 \\
\hline Callorhinchus callorynchus & & & 2,3 & 97,7 & & & 0,7 & 99,3 & & 3,4 & 15,5 & 81,1 & & & 2,9 & 97,0 \\
\hline Calyptraster sp. & & & & & & & & 100 & & & & & & & & \\
\hline Campylonotus vagans & & & & 100 & & & & & & & & & & & & 100 \\
\hline Congiopodus peruviamus & & & & 100 & & & & 100 & & & & 100 & & & & 100 \\
\hline Cottoperca trigloides & & & 20,0 & 80,0 & & & & 100 & & & & 100 & & & & 100 \\
\hline Cynoscion guatucupa & & & & & & & & 100 & & & & & & & & 100 \\
\hline Diplasterias brandti & & & & & & & & & & & & & & & & 100 \\
\hline Dipturus argentinensis & & & & & & & & & & & & 100 & & & & 100 \\
\hline Dipturus trachyderma & & & & & & & & & & & & 100 & & & & 100 \\
\hline Discopyge tschudii & & & & 100 & & & & 100 & & & & 100 & & & & 99 \\
\hline Eleginops maclovimus & & & & 100 & & & & 100 & & & & 100 & & & & 100 \\
\hline Engraulis anchoita & & & & 100 & & & & 100 & & & & 100 & & & 5,2 & 94 \\
\hline Eurypodius latreillei & & & & & & & & 100 & & & & & & & & 100 \\
\hline Galeorhinus galeus & & & & 100 & & & & 100 & & & & 100 & & & & 100 \\
\hline Genypterus blacodes & & & & 99,7 & & & & 100 & & & 3,0 & 97,0 & & & & 99,6 \\
\hline Genypterus brasiliensis & & & & 100 & & & & 100 & & & & 100 & & & & 100 \\
\hline Illex argentimus & & & & 100 & & & & 100 & & & & 95 & & 0,2 & 2,4 & 60 \\
\hline Leurocyclus tuberculosus & & & & & & & & 100 & & & & 100 & & & & 100 \\
\hline Libidoclaea granaria & & 1,3 & 1,3 & 97,5 & & & & 100 & & & 2,1 & 97,9 & & & & 100 \\
\hline Libinia spinosa & & & & & & & & 100 & & & & & & & & 100 \\
\hline Lithodes santolla & 1,7 & 1,7 & 17,0 & 79,6 & & 0,3 & 1,7 & 98,0 & & & 1,9 & 98,1 & 0,2 & & 7,4 & 94,9 \\
\hline Loligo spp. & & & & 100 & & & 0,5 & 100 & & & & 100 & & & 1,3 & 99 \\
\hline Macruronus novaezelandiae & & & & & & & & 100 & & & & 100 & & & & 100 \\
\hline Magallanica venosa & & & & & & & & 100 & & & & & & & & \\
\hline Merluccius hubbsi & 38,6 & 28,9 & 24,0 & 8,5 & 24,9 & 28,4 & 10,9 & 35,8 & 99,7 & 1,9 & & 0,5 & 93,5 & 4,6 & 0,2 & 0,9 \\
\hline Mullus argentinae & & & & & & & & & & & & & & & & 100 \\
\hline Munida gregaria & 8,9 & 20,0 & 2,2 & 68,9 & 7,8 & 3,2 & 19,2 & 69,9 & & & 15,2 & 84,8 & 0,4 & 7,7 & 9,8 & 82,2 \\
\hline Mustelus schmitti & & & & 100 & & & & 100 & & & 2,6 & 97 & & & & 100 \\
\hline Myliobatis goodei & & & & 100 & & & & 100 & & & & 100 & & & & 100 \\
\hline Mytilus edulis platensis & & & & & & & & 100 & & & & & & & & \\
\hline Myxine australis & & & & 100 & & & & 100 & & & & 100 & & & & 100 \\
\hline Nemadactylus bergi & & & & 100 & & & 7,4 & 92,6 & & & & 100 & & & & 100 \\
\hline Notorhynchus cepedianus & & & & & & & & & & & & 100 & & & & 100 \\
\hline
\end{tabular}




\begin{tabular}{|c|c|c|c|c|c|c|c|c|c|c|c|c|c|c|c|c|}
\hline & \multicolumn{8}{|c|}{ Langostino } & \multicolumn{8}{|c|}{ Merluza } \\
\hline & \multicolumn{4}{|c|}{ Área centro } & \multicolumn{4}{|c|}{ Área norte } & \multicolumn{4}{|c|}{ Área centro } & \multicolumn{4}{|c|}{ Área norte } \\
\hline & Do & $\mathrm{Ab}$ & Co & $\mathrm{Ra}$ & Do & $\mathrm{Ab}$ & Co & $\mathrm{Ra}$ & Do & $\mathrm{Ab}$ & Co & $\mathrm{Ra}$ & Do & $\mathrm{Ab}$ & Co & $\mathrm{Ra}$ \\
\hline Odontesthes smitti & & & & 100 & & & & 100 & & & & & & & & 100 \\
\hline Ovalipes trimaculatus & & & & & & & & 100 & & & & & & & & 100 \\
\hline Propagurus gaudichauidi & & & & & & & & & & & & & & & & 100 \\
\hline Paralichthys isosceles & & & & 100 & & & & 100 & & & & 100 & & & & 100 \\
\hline Paralichthys patagonicus & & & & & & & & 100 & & & & & & & & 100 \\
\hline Parona signata & & & & & & & & 100 & & & 2,0 & 94,1 & & 1,7 & 2,7 & 95,5 \\
\hline Patagonotothen ramsayi & & & & & & & & 100 & & 1,5 & 4,2 & 94,2 & & & 1,6 & 99,0 \\
\hline Peisos petrunkevitchi & & & & 100 & & & 13,6 & 86 & & & & 20 & 3,7 & 3,7 & 2,2 & 90 \\
\hline Peltarion spinosulum & & & & 100 & & & & 81 & & & & 83 & & & & 100 \\
\hline Percophis brasiliensis & & & & & & & & & & & & & & & & 100 \\
\hline Pinguipes brasilianus & & & & & & & & 100 & & & & & & & & 100 \\
\hline Platyxhantus patagonicus & & & & 100 & & & & 100 & & & & 100 & & & & \\
\hline Pleoticus muelleri & 27,2 & 41,9 & 19,0 & 11,9 & 40,4 & 26,8 & 14,1 & 14,3 & & 0,7 & 7,9 & 91,4 & 1,3 & 0,4 & 1,6 & 96,6 \\
\hline Polyprion americanus & & & & & & & & & & & & 100 & & & & 100 \\
\hline Pontocaris boschii & & & & & & & & & & & & & & & & 100 \\
\hline Prionotus nudigula & & & & & & & & & & & & & & & & 100 \\
\hline Psammobatis lentiginosa & & & & & & & & 100 & & & & 100 & & & & 37,5 \\
\hline Psammobatis normani & & & & 100 & & & & 100 & & & 0,8 & 100 & & & & 100 \\
\hline Psammobatis rudis & & & & 100 & & & & 100 & & & & 100 & & & & 100 \\
\hline Pseudopercis semifasciata & & & & 100 & & & & 100 & & & & 100 & & & & 100 \\
\hline Pterygosquilla armata armata & & & & 100 & & & & 100 & & & & 100 & & & & 100 \\
\hline Raneya brasiliensis & & & & 100 & & & & 100 & & & & & & & & 100 \\
\hline Renilla sp, & & & & 100 & & & 17,7 & 82 & & & & 100 & & & 2,1 & 98 \\
\hline Salilota australis & & & & 100 & & & & 100 & & & & 100 & & & & 100 \\
\hline Schroederichthys bivius & & & & 100 & & & & 100 & & & & 100 & & & & 100 \\
\hline Scomber japonicus & & & & & & & & & & & & & & & & 100 \\
\hline Sebastes oculatus & & & & 100 & & & & 100 & & & & 100 & & & & 100 \\
\hline Semirossia tenela & & & & & & & & 100 & & & & & & & & \\
\hline Seriolella porosa & & & & 100 & & & & 100 & & & & 100 & & 0,7 & 3,0 & 96 \\
\hline Serolis spp, & & & & & & & & 100 & & & & & & & & \\
\hline Squalus acanthias & & & & 100 & & & & 100 & & 0,9 & 3,0 & 96 & & & & 100 \\
\hline Squalus mitsukurii & & & & & & & & & & & & 100 & & & & 100 \\
\hline Squatina guggenheim & & & & & & & & 100 & & & & 100 & & & & 100 \\
\hline Stromateus brasiliensis & & & & 100 & & & 1,5 & 99 & & & 0,5 & 100 & & & 1,1 & 98 \\
\hline Sympterygia bonapartii & & 0,7 & 1,4 & 97,9 & & & & 100 & & & 3,9 & 96,1 & & & & 99,6 \\
\hline Torpedo puelcha & & & & & & & & & & & & & & & & 100 \\
\hline Triathalassothia argentina & & & & & & & & 100 & & & & 100 & & & & \\
\hline Tritonia adheneri & & & & 100 & & & & 100 & & & & 100 & & & & 100 \\
\hline Trypilaster $\mathrm{sp}$ & & & & & & & & & & & & & & & & 100 \\
\hline Urophycis brasiliensis & & & & & & & & & & & & & & & & 100 \\
\hline Xystreurys rasile & & & & 100 & & & & 100 & & & & 101 & & & & 100 \\
\hline Zearaja chilensis & & & & 100 & & & & 100 & & 0,6 & 9,3 & 90 & & & 3,4 & 97 \\
\hline
\end{tabular}

$10 \%$ es un valor débil si la tabla contiene 10 variables y es un valor fuerte en el caso de 100 variables, como es el caso de la tabla área $\times$ especie usada en este análisis.

El agrupamiento de los sitios se apoya en el conjunto de las variables (especies) de todos los años considerados en el presente trabajo. En la Figura 2 se observa la distribución en el plano formado por los factores 1 y 2 de los sitios que contribuyeron al modelo en un porcentaje mayor al 1\%. Los mismos se ordenaron en 2 grandes agrupamientos que están asociados a su vez a la especie objetivo, por un lado se distingue el norte del Golfo San Jorge cuando la flota operó sobre merluza, y las áreas del centro del golfo y norte juntas cuando la especie objetivo es el langostino. Hay 2 áreas muy cercanas a la costa en 


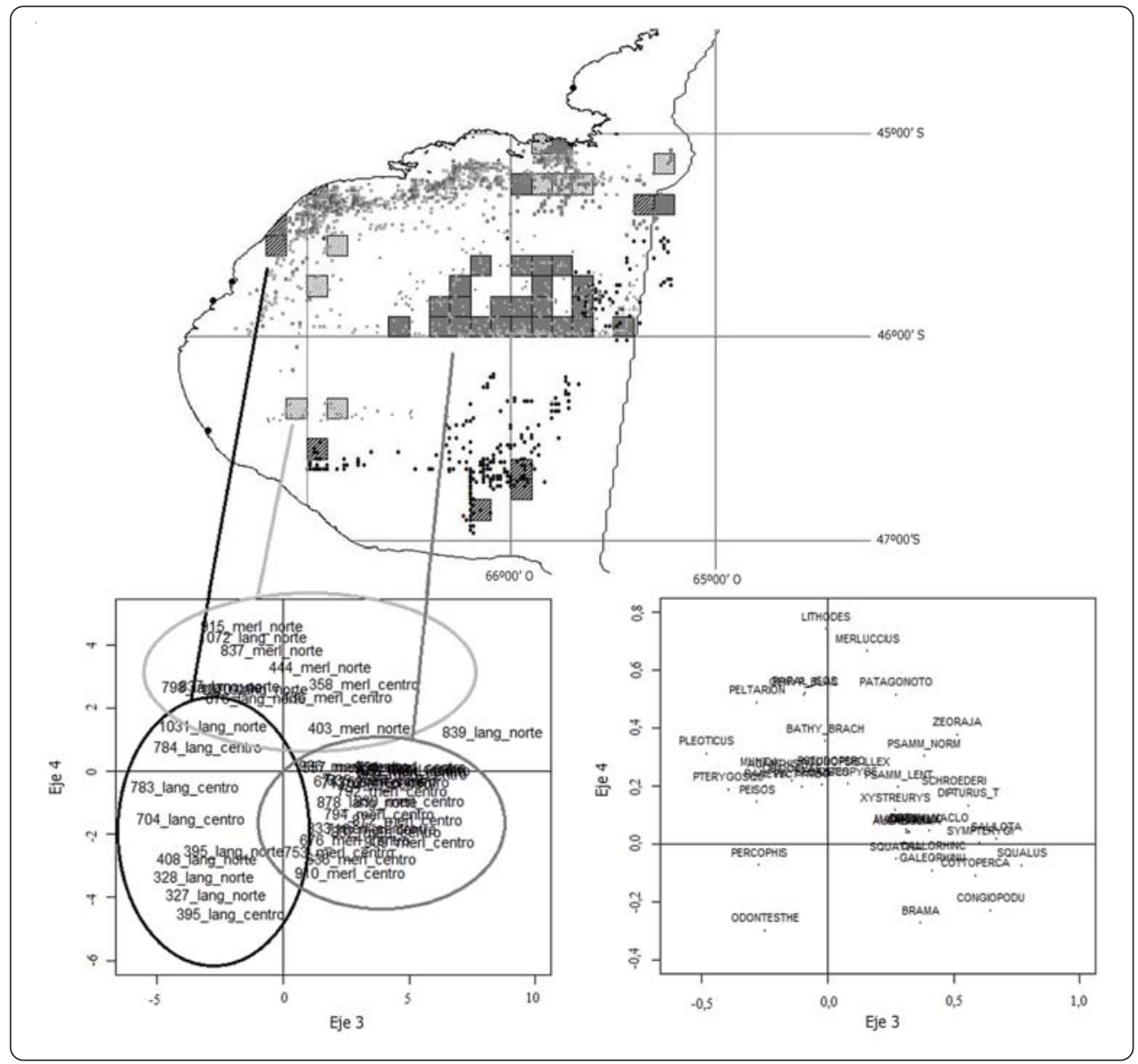

Figura 3. Análisis de componentes principales: áreas (individuos) que contribuyen a los factores (3 y 4) y representación de las variables. Para mejor visualización se presentan sólo los puntos, se eliminaron las líneas que indicaban los vectores y círculo de correlación / Principal component analysis: areas (individuals) that contribute to factors ( 3 and 4 ) and representation of variables. To improve visualization only points are showed, lines were removed indicating vectors and the correlation circle

el norte del Golfo San Jorge en una zona conocida como Pan de Azúcar, que cuando la especie objetivo es langostino identifican claramente el eje 1 presentando las coordenadas más extremas.

Cuando la flota operó en el norte del golfo y la especie objetivo fue la merluza, los taxones (variables) que caracterizaron esta zona y cuya contribución a los factores 1 y 2 fue superior al 1\% fueron los peces: Callorhinchus callorynchus, Atlantoraja castelnaui, Parona signata, Pseudopercis semifasciata, Acantisthius patachonicus, Stromateus brasiliensis, Engraulis anchoita, Eleginops maclovinus y Sebastes oculatus y los crustáceos: Ovalipes trimaculatus y Munida gregaria.
Hay 2 grupos de especies que no se relacionaron claramente con las áreas descritas: el primero compuesto por Squalus acanthias, Zearaja chilensis y Schroederichthys bivius, más cercano a los rectángulos del centro del golfo y el segundo compuesto por Psammobatis lentiginosa, Squatina guggenheim, Nemadactylus bergi, Paralichthys patagonicus, Renilla sp. y Eurypodius latreillei grupo más cercano a las áreas del norte del golfo.

En la Figura 3 se observa la distribución en el plano formado por los factores 3 y 4 de los sitios que contribuyeron al modelo en un porcentaje mayor al $1 \%$, la distribución de los ejes 1 y 3, y 2 y 3 repetía los 
agrupamientos de los ejes 1 y 2, por ello se presentan los factores 3 y 4 donde se observan 3 grandes agrupamientos, en 2 de ellos el patrón no es claro, un agrupamiento tiene como características común la especie objetivo langostino pero no las áreas, un segundo agrupamiento mezcla especie objetivo merluza y langostino y no hay un área definida, por último un tercer agrupamiento reúne los rectángulos del centro del Golfo San Jorge cuando la especie objetivo es merluza. Este último agrupamiento es el más interesante caracterizado por Callorhinchus callorynchus, Squalus acanthias, Galeornihus galeus, Squatina guggenheim, Brama brama, Congiopodus peruvianus, Cottoperca trigloides y Salilota australis.

\section{Discusión}

En la flota fresquera de altura que operó en el Golfo San Jorge en el período 2003 a 2012 se identificaron en las capturas un total de 90 taxones, 33 invertebrados, 35 peces óseos y 22 peces cartilaginosos. Cuando la flota estuvo dirigida a merluza se identificaron 79 taxones en el área norte del Golfo San Jorge y 60 en el centro del golfo. Cuando la flota operó dirigida a langostino se identificaron 71 taxones en el norte y 50 en el centro del golfo. Las especies Z. chilensis, M. hubbsi, S. brasiliensis, P. ramsayi, G. blacodes, I. argentinus, Loligo spp. y L. santolla presentaron una FO superior al $40 \%$ en todas las áreas e indistintamente de la especie objetivo.

Góngora et al. (2009) describieron la captura incidental de la flota congeladora tangonera cuya especie objetivo es el langostino, y cuya área fue el Golfo San Jorge en su conjunto, identificando en sus capturas un total de 68 especies de peces (44 especies de peces óseos, 20 especies de peces cartilaginosos y 2 especies de mixines), siendo esta superior al número de especies de peces registrados en las capturas de la flota fresquera de altura, aun cuando dicha flota operó sobre langostino.

Cabe destacar la presencia en las capturas de la flota fresquera de altura de las especies Cynoscion guatucupa, Prionotus nudigula, Urophycis brasiliensis, Mullus argentinae, Atlantorraja castelnaui y Atlantorraja cyclophora que fueron registradas recientemente en el Golfo San Jorge (Bovcon et al. 2011).

En los análisis multivariados se observan 2 agrupamientos, el primero cuando la especie objetivo fue la merluza y el otro cuando la especie objetivo fue el langostino. Cuando la especie objetivo fue la merluza la composición de las capturas varió según las áreas de pesca pero este patrón no se observó cuando la especie objetivo fue el langostino. No obstante, en los análisis cualitativos de descripción de los taxones se observó una mayor riqueza en las capturas cuando la flota fresquera operó en el norte del Golfo San Jorge sobre langostino versus el centro del golfo San Jorge sobre langostino (71 vs 50 taxones respectivamente), aunque las especies dominantes son las mismas en las 2 áreas.

El norte del Golfo San Jorge se caracterizó por la presencia de peces de arrecifes rocosos o asociados a estos ambientes (Pseudopercis semifasciata, Sebastes oculatus, Pinguipes brasilianus, Eleginops maclovinus y Nemadactylus bergi), peces costeros y demersalpelágicos (Odontesthes smitti, Seriolella porosa, Stromateus brasiliensis y Parona signata) y algunos invertebrados (Munida gregaria, Renilla sp., Peltarion spinosulum, Peisos petrunkevitchi y Aphrodita longicornis), tanto en el análisis multivariado como en el análisis cualitativo. Los peces de roca fueron identificados ya por Góngora (2011) a nivel cualitativo. En el presente trabajo se lo complementa en forma cuantitativa, ya que este grupo de especies caracterizó el norte del Golfo San Jorge cuando la especie objetivo fue la merluza. Góngora (2011) los observó en el norte del Golfo en la flota congeladora tangonera que operó sobre langostino.

En tanto el centro del Golfo San Jorge estuvo caracterizado por especies de aguas profundas, Squalus acanthias, Galeorhinus galeus, Squatina guggenheim, Brama brama, Congiopodus peruvianus, Cottoperca trigloides, Salilota australis, Zearaja chilensis y Schroederichthys bivius.

La zona costera del norte del Golfo San Jorge también fue identificada como área de asociaciones de especie por Góngora (2011) a partir de información de la captura incidental de la flota congeladora tangonera que tiene como especie objetivo el langostino. En este trabajo la zona fue caracterizada por especies costeras como Nemadactilus bergi, Engraulis anchoita, Odontesthes smitti, Eleginops maclovinus, Squatina guggenheim, Pseudopercis semifasciata, Parona signata y Paralichthys patagonicus.

Distintos factores podrían interactuar y determinar que el norte sea un área diferente del centro del Golfo San Jorge. Esta diferencia en la diversidad del norte con el centro del Golfo San Jorge, indistintamente de cuál fuese la especie objetivo, puede ser resultado de la proximidad a la costa, menor profundidad y la heterogeneidad de hábitats que presenta el área. Otro factor que puede estar contribuyendo es la corriente de Brasil, que según López 
Tabla 5. Frecuencia de ocurrencia por categoría de destino, área y especie objetivo: encajonada (En), parcialmente encajonada (Pe), descartada (De) / Frequency of occurrence by destination category, area and target species: boxed in (En), partially encased (Pe), discarded (De)

\begin{tabular}{|c|c|c|c|c|c|c|c|c|c|c|c|c|}
\hline & \multicolumn{6}{|c|}{ Langostino } & \multicolumn{6}{|c|}{ Merluza } \\
\hline & \multicolumn{3}{|c|}{ Área centro } & \multicolumn{3}{|c|}{ Área norte } & \multicolumn{3}{|c|}{ Área norte } & \multicolumn{3}{|c|}{ Área centro } \\
\hline & De & $\mathrm{Pe}$ & En & $\mathrm{De}$ & $\mathrm{Pe}$ & En & De & $\mathrm{Pe}$ & En & $\mathrm{De}$ & $\mathrm{Pe}$ & En \\
\hline Acanthistius patachonicus & 100 & & & 100 & & & 64,2 & 11,1 & 24,7 & 44,0 & 12,0 & 44,0 \\
\hline Acodontaster sp, & & & & 100 & & & & & & & & \\
\hline Aequipecten tehuelchus & & & & 100 & & & & & & & & \\
\hline Agonopsis chiloensis & 100 & & & 100 & & & & & & & & \\
\hline Pitaria rostrata & & & & 100 & & & & & & & & \\
\hline Aphrodita longicornis & & & & 100 & & & 100 & & & & & \\
\hline Arbacia dufresnei & & & & 100 & & & & & & & & \\
\hline Artemesia longinaris & & & & & & & 100 & & & & & \\
\hline Atlantoraja castelnaui & & & & & & & 68,6 & 14,3 & 17,1 & 33,3 & 33,3 & 33,3 \\
\hline Atlantoraja cyclophora & & & & & & & 47,6 & 14,3 & 38,1 & 92,3 & & 7,7 \\
\hline Aulacomya ater & & & & 100 & & & & & & & & \\
\hline Austrolycus laticinctus & & & & & & & & & & 25,0 & & \\
\hline Bathyraja brachyurops & & & & & & & 95,5 & & 0,2 & 100 & & \\
\hline Bathyraja macloviana & 100 & & & 100 & & & 64,9 & & 35,1 & 74,2 & 25,8 & \\
\hline Brama brama & 0,0 & 100 & & & & & 72,7 & & 27,3 & 39,3 & 14,3 & 46,4 \\
\hline Callorhinchus callorynchus & 88,6 & 2,3 & 9,1 & 78,6 & 13,1 & 8,3 & 21,7 & 19,1 & 59,2 & 13,0 & 13,9 & 73,1 \\
\hline Calyptraster sp, & & & & 100 & & & & & & & & \\
\hline Campylonotus vagans & 100 & & & & & & 100 & & & & & \\
\hline Congiopodus peruvianus & 100 & & & 100 & & & 100 & & & 100 & & \\
\hline Cottoperca trigloides & 100 & & & 100 & & & 100 & & & 100 & & \\
\hline Cynoscion guatucupa & & & & 100 & & & & & & & & \\
\hline Diolasterias brandti & & & & & & & 100 & & & & & \\
\hline \multicolumn{13}{|l|}{ Dipturus argentinensis } \\
\hline Dipturus trachyderma & & & & 100 & & & 9,6 & 27,0 & 62,6 & 21,3 & 47,9 & 30,9 \\
\hline Discopyge tschudii & 100 & & & 100 & & & 100 & & & 100 & & \\
\hline Eleginops maclovinus & 100 & & & 90,9 & & 9,1 & 30,1 & 12,6 & 57,3 & 40,0 & 40,0 & 20,0 \\
\hline Engraulis anchoita & 100 & & & 100 & & & 97,4 & 1,3 & 1,3 & & & \\
\hline Eurypodius latreillei & & & & 100 & & & 100 & & & & & \\
\hline Galeorhinus galeus & 100 & & & 100 & & & 82,7 & & 17,3 & 25,3 & 7,6 & 67,1 \\
\hline Genypterus blacodes & 93,2 & 4,5 & 2,3 & 77,8 & 16,0 & 6,2 & 57,5 & 18,4 & 23,7 & 58,2 & 13,4 & 28,4 \\
\hline Genypterus brasiliensis & 100 & & & 50,0 & 50,0 & & 48,3 & 34,5 & 17,2 & 63,6 & & 36,4 \\
\hline Illex argentinus & 99,3 & & 0,7 & 79,1 & 7,4 & 13,5 & 41,2 & 31,7 & 27,1 & 24,0 & 29,8 & 46,2 \\
\hline Leurocyclus tuberculosus & & & & 100 & & & 100 & & & 100 & & \\
\hline Libidoclaea granaria & 100 & & & 100 & & & 100 & & & 99,3 & 0,7 & \\
\hline Libinia spinosa & & & & 100 & & & 100 & & & & & \\
\hline Lithodes santolla & 38,5 & 60,3 & 1,1 & 53,7 & 46,0 & 0,3 & 39,8 & 51,9 & 11,0 & 52,5 & 40,0 & 7,5 \\
\hline Loligo spp. & 97,3 & 2,7 & & 96,8 & 2,3 & 0,9 & 59,8 & 36,3 & 3,9 & 92,4 & 6,5 & \\
\hline Macruronus novaezelandiae & & & & 100 & & & & & 100 & 78,6 & & \\
\hline Magallanica venosa & & & & 100 & & & & & & & & \\
\hline Merluccius hubbsi & 73,8 & 26,2 & & 73,5 & 26,5 & & 0,4 & 91,4 & 8,1 & 0,5 & 83,9 & \\
\hline Mullus argentinae & & & & 100 & & & 100 & & & & & \\
\hline Munida gregaria & 100 & & & 100 & & & 100 & & & 100 & & \\
\hline Mustelus schmitti & 100 & & & 100 & & & 83,7 & & 15,1 & 76,3 & & 23,7 \\
\hline Myliobatis goodei & 100 & & & 100 & & & 91,7 & & 8,3 & 100 & & \\
\hline Mytilus edulis platensis & & & & 100 & & & & & & & & \\
\hline Myxine australis & 100 & & & 100 & & & 100 & & & 100 & & \\
\hline Nemadactylus bergi & 100 & & & 100 & & & 100 & & & 100 & & \\
\hline Notorhynchus cepedianus & & & & & & & & & 100 & 100 & & \\
\hline
\end{tabular}




\begin{tabular}{|c|c|c|c|c|c|c|c|c|c|c|c|c|}
\hline & \multicolumn{6}{|c|}{ Langostino } & \multicolumn{6}{|c|}{ Merluza } \\
\hline & \multicolumn{3}{|c|}{ Área centro } & \multicolumn{3}{|c|}{ Área norte } & \multicolumn{3}{|c|}{ Área norte } & \multicolumn{3}{|c|}{ Área centro } \\
\hline & $\mathrm{De}$ & $\mathrm{Pe}$ & En & $\mathrm{De}$ & $\mathrm{Pe}$ & En & $\mathrm{De}$ & $\mathrm{Pe}$ & En & $\mathrm{De}$ & $\mathrm{Pe}$ & En \\
\hline Odontesthes smitti & 100 & & & 100 & & & & & 50,0 & & & \\
\hline Ovalipes trimaculatus & & & & 100 & & & 100 & & & & & \\
\hline Propagurus gaudichauidi & & & & & & & 100 & & & & & \\
\hline Paralichthys isosceles & 100 & & & 96,9 & 3,1 & & 86,1 & & 6,9 & 20,8 & 66,7 & 12,5 \\
\hline Paralichthys patagonicus & & & & 100 & & & 78,6 & & 7,1 & & & \\
\hline Parona signata & & & & 81,3 & 12,5 & 6,3 & 41,2 & 24,1 & 34,7 & 23,5 & & 68,6 \\
\hline Patagonotothen ramsayi & & & & 100 & & & 99,7 & & 0,6 & 99,6 & 0,4 & \\
\hline Peisos petrunkevitchi & 100 & & & 100 & & & 100 & & & 100 & & \\
\hline Peltarion spinosulum & 100 & & & 100 & & & 100 & & & 100 & & \\
\hline Percophis brasiliensis & & & & & & & 70,0 & & & & & \\
\hline Pinguipes brasiliamus & & & & 100 & & & 100 & & & & & \\
\hline Platyxanthus patagonicus & 100 & & & 100 & & & & & & & & \\
\hline Pleoticus muelleri & 0,8 & 41,4 & 57,8 & 5,0 & 23,2 & 70,0 & 24,6 & 31,6 & 43,8 & 29,3 & 32,1 & 38,6 \\
\hline Polyprion americanus & & & & & & 100 & 33,3 & & 58,3 & & & \\
\hline Pontocaris boschii & & & & & & & 100 & & & & & \\
\hline Prionotus nudigula & & & & & & & 100 & & & & & \\
\hline Psammobatis lentiginosa & & & & 100 & & & 100 & & & 100 & & \\
\hline Psammobatis normani & 100 & & & 100 & & & 91,4 & 5,4 & 3,2 & 86,9 & 12,7 & \\
\hline Psammobatis rudis & 100 & & & 100 & & & 100 & & & 100 & & \\
\hline Pseudopercis semifasciata & 100 & & & 61,5 & 7,7 & 30,8 & 52,2 & 13,9 & 33,9 & 76,7 & & 23,3 \\
\hline Pterygosquilla armata armata & 100 & & & 100 & & & 100 & & & 100 & & \\
\hline Raneya brasiliensis & 100 & & & 100 & & & & & & & & \\
\hline Renilla sp. & 100 & & & 100 & & & 100 & & & 98,8 & 1,2 & \\
\hline Salilota australis & 100 & & & 100 & & & 93,2 & & & 95,0 & 2,0 & \\
\hline Schroederichthys bivius & 100 & & & 100 & & & 100 & & & 100 & & \\
\hline Scomber japonicus & & & & & & & & & 100 & & & \\
\hline Sebastes oculatus & 87,5 & 12,5 & & 100 & & & 100 & & & 86,4 & 9,1 & \\
\hline Semirossia tenela & & & & 100 & & & & & & & & \\
\hline Seriolella porosa & 100 & & & 80,0 & 13,3 & 6,7 & 44,8 & 9,4 & 45,8 & 31,8 & & 40,9 \\
\hline Serolis spp. & & & & 100 & & & & & & & & \\
\hline Squalus acanthias & 100 & & & 100 & & & 93,8 & & 5,4 & 97,8 & & 1,3 \\
\hline Squalus mitsukurii & & & & & & & 100 & & & 100 & & \\
\hline Squatina guggenheim & & & & 100 & & & & & & 100 & & \\
\hline Stromateus brasiliensis & 100 & & & 100 & & & 99,4 & & 0,3 & 99,5 & 0,5 & \\
\hline Sympterygia bonapartii & 100 & & & 100 & & & 57,7 & 18,3 & 53,7 & 27,3 & 51,2 & 21,5 \\
\hline \multicolumn{13}{|l|}{ Torpedo puelcha } \\
\hline Triathalassothia argentina & & & & 100 & & & & & & 100 & & \\
\hline Tritonia adheneri & 100 & & & 100 & & & 100 & & & & & \\
\hline Trypilaster $s p$ & & & & & & & 100 & & & & & \\
\hline Urophycis brasiliensis & & & & & & & 100 & & & & & \\
\hline Xystreurys rasile & 74,0 & 23,4 & 2,6 & 88,9 & 11,1 & & 81,9 & 16,6 & 1,2 & 56,9 & 32,2 & 11,5 \\
\hline Zearaja chilensis & 100 & & & 100 & & & 27,8 & 21,4 & 50,7 & 5,1 & 61,4 & 33,4 \\
\hline
\end{tabular}


Tabla 6. Análisis de Componentes Principales. Autovalores y porcentaje de inercia explicado por cada factor / Principal component analysis. Eigenvalues and percentage of inertia explained by factor

\begin{tabular}{lrrc}
\hline & Autovalores & \% inercia & $\begin{array}{c}\text { \% inercia } \\
\text { acumulada }\end{array}$ \\
\hline Factor 1 & 9,865 & 13,894 & 13,89 \\
Factor 2 & 7,017 & 9,883 & 23,78 \\
Factor 3 & 6,210 & 8,747 & 32,52 \\
Factor 4 & 3,743 & 5,272 & 37,80 \\
\hline
\end{tabular}

(1964) puede llegar en el verano hasta el Golfo San Jorge. Esta misma observación fue realizada por Balech (1971) quien registró transgresiones de la corriente de Brasil que llegaban hasta la Isla Leones $\left(45^{\circ} 03^{\prime} \mathrm{S}\right.$; $\left.65^{\circ} 37^{\prime} \mathrm{O}\right)$ y Cabo Blanco $\left(47^{\circ} 12^{\prime} \mathrm{S} ; 65^{\circ} 45^{\prime} \mathrm{O}\right)$, cuya intensidad y duración fue fluctuante en cada año. Según Balech (1971) es indiscutible la presencia de organismos nectónicos y bentónicos brasileños en el sur del Golfo San Jorge ya que la distribución de los organismos bentónicos se hace por medio de larvas planctónicas.

Algunos taxones sólo fueron registrados en el norte del golfo con frecuencias muy bajas: Urophycis brasiliensis, Mullus argentinae, Prionotus nudigula, Cynoscion guatucupa, Paralichthys patagonicus, Semirossia tenela, Acodontaster sp. y Calyptraster sp. Mientras que en el centro del golfo solo se registró Austrolycus laticinctus.

El norte del Golfo San Jorge es un área relevante en términos de biodiversidad y productividad. Se caracteriza por una alta diversidad de ambientes costeros y marinos, los cuales sirven de áreas de reproducción y cría a distintas especies de peces, invertebrados, aves, mamíferos marinos y áreas de alimentación y descanso

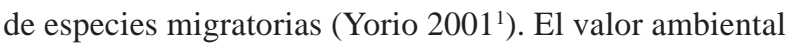
del sector norte de este golfo motivó la creación de un área protegida costera de $750 \mathrm{~km}^{2}$, administrada en forma conjunta por la Administración de Parques Nacionales y el Gobierno de la Provincia del Chubut (Ley Nacional 26446/2008) (Yorio 2009). Este parque marino sólo se extiende 1 milla náutica de la línea de costa, no llegando a cubrir las áreas de pesca del norte del Golfo San Jorge.
En relación a la abundancia en las capturas, la merluza es siempre dominante o abundante, $Z$. chilensis fue común en ambos lugares, $M$. gregaria fue abundante y común en el norte del golfo, C. callorynchus y P. ramsayi sólo son comunes y/o abundantes en el centro del golfo.

En la flota fresquera de altura, el aprovechamiento es integral cuando la flota operó sobre merluza. Sin embargo, cuando la flota operó sobre langostino se descartaron la mayoría de los taxones, debido al alto valor de esta especie objetivo. Cuando la especie objetivo fue la merluza se aprovecharon entre el 50 y el $60 \%$ de los taxones capturados, 11 taxones se aprovecharon en más de un $60 \%$ de los lances en los que la especie fue capturada. Cuando la especie objetivo fue el langostino se aprovechó sólo el 20\% de los taxones, y 3 o 4 especies fueron desembarcadas, entre ellas la más importante fue centolla.

\section{Agradecimientos}

A la Secretaría de Pesca de la Provincia del Chubut, especialmente al Programa de Observadores a Bordo, al Centro Nacional Patagónico (CONICET) a la Universidad Nacional de la Patagonia San Juan Bosco y a la Fundación Rufford.

\section{LITERATURA CITADA}

Balech E. 1971. Notas históricas y críticas de la oceanografía biológica argentina. Servicio de Hidrografía Naval, Buenos Aires, H1027: 1-57.

Bezzi S \& C Dato. 1995. Conocimiento biológico pesquero del recurso merluza (Merluccius hubbsi) y su pesquería en la República Argentina. Documento Científico 4: 3-52. Instituto de Investigación y Desarrollo Pesquero, Mar del Plata.

Bezzi S, M Renzi, G Irusta, B Santos, L Tringali, M Ehrlich, F Sánchez, S García de la Rosa, M Simonazzi \& R Castrucci. 2004. Caracterización biológica y pesquera de la merluza (Merluccius hubbsi). En: Sánchez R \& S Bezzi (eds). El Mar Argentino y sus recursos pesqueros. Tomo 4. Los peces marinos de interés pesquero. Caracterización biológica y evaluación del estado de explotación, pp. 157206. Instituto de Investigación y Desarrollo Pesquero, Mar del Plata.

Bovcon ND, PD Cochia, ME Góngora \& AE Gosztonyi. 2011. Records of warm-temperate water fishes in central Patagonian coastal waters (Southwestern South Atlantic Ocean). Journal of Applied Ichthyology 27: 832-839.

\footnotetext{
${ }^{1}$ Yorio P. 2001. Justificativos para la creación de una nueva área marina protegida en la Provincia de Chubut: el norte del golfo San Jorge. Proyecto 'Consolidación e implementación del Plan de Manejo de la Zona Costera Patagónica para la conservación de la biodiversidad'. Proyecto GEF/PNUD (ARG/ 97/ G31). Ministerio de Relaciones Exteriores, Comercio Internacional y Culto.
} 
Cordo H. 2006. Evaluación del estado del efectivo sur de $41^{\circ} \mathrm{S}$ de la merluza (Merluccius hubbsi) y estimación de la captura biológicamente aceptable correspondiente al año 2006. Informe Técnico Interno 34/06: 1-27. Instituto de Investigación y Desarrollo Pesquero, Mar del Plata.

Crawley MJ. 2007. The R book, 942 pp. John Wiley \& Sons, West Sussex.

Dato C, G Bambill, G Cañete, MF Villarino \& A Aubone. 2006. Estimación cuantitativa del descarte en la pesquería de merluza realizado por la flota comercial argentina. Documento Científico 6: 31-38. Instituto de Investigación y Desarrollo Científico, Mar del Plata.

Eschmeyer WN. 2013. Catalog of fishes. California Academy of Sciences, San Francisco. <http://research.calacademy.org/ research/ichthyology/catalog/fishcatmain.asp>

Escofier B \& J Pagès. 1992. Análisis factoriales simples y múltiples. Objetivos, métodos e interpretación, 285 pp. Universidad del País Vasco, Leioa.

Gauch HG. 1989. Multivariate analysis in community ecology, 299 pp. Cambridge University Press, Cambridge.

Góngora ME. 2011. Dinámica y manejo de la captura incidental de peces en la pesquería de langostino patagónico (Pleoticus muelleri). Tesis doctoral, Centro Regional Universitario Bariloche, Universidad Nacional del Comahue, Bariloche, 214 pp.

Góngora ME, ND Bovcon \& PD Cochia. 2009. Ictiofauna capturada incidentalmente en la pesquería de langostino patagónico Pleoticus muelleri Bate, 1888 (Solenoceridae). Revista de Biología Marina y Oceanografía 44(3): 583-593.

Góngora ME, D Gónzalez-Zevallos, A Pettovello \& L Mendia. 2012. Caracterización de las principales pesquerías del golfo San Jorge Patagonia, Argentina. Latin American Journal of Aquatic Research 40(1): 1-11.

Hall M. 1996. On bycatch. Reviews in Fish Biology and Fisheries 6: 319-352.
Hall M, DL Alverson \& K Metuzals. 2000. By-catch: problems and solutions. Marine Pollution Bulletin 41(16): 204-219.

Kelleher K. 2008. Descartes en la pesca de captura marina mundial. FAO Documento Técnico de Pesca 470: 1-147.

Lê S, J Josse \& F Husson. 2008. Facto miner: An R package for multivariate analysis. Journal of Statistical Software 25(1): 1-18.

Legendre P \& L Legendre. 1998. Numerical ecology. Developments Environmental Modelling 20: 1-853. Elsevier Science B.V, Amsterdam.

López RB. 1964. Problemas de la distribución geográfica de los peces marinos Suramericanos. Boletín, Instituto de Biología Marina, Mar del Plata 7: 57-99.

Pettovello A. 1999. Bycatch in the Patagonian red shrimp (Pleoticusmuelleri) fishery. Marine and Freshwater Research 50: 123-127.

R Development Core Team. 2006. R: A language and environmental for statistical computing. R Foundation for Statistical Computing, Viena. < http://www.R-project.org>

Renzi MA, MF Villarino \& B Santos. 2009. Evaluación del estado de explotación del efectivo sur de $41^{\circ} \mathrm{S}$ de la merluza (Merluccius hubbsi) y estimación de las capturas biológicamente aceptables correspondientes al año 2009 y 2010. Informe Técnico Interno 46/09: 1-37. Instituto Nacional de Investigación y Desarrollo Pesquero, Mar del Plata.

Stobutzki IC, P Jones \& M Miller. 2003. A comparison of fish bycatch communities between areas open and closed to prawn trawling in an Australian tropical fishery. ICES Journal of Marine Science 60: 951-966.

Yorio P. 2009. Marine protected areas, spatial scales, and governance: implications for the conservation of breeding seabirds. Conservation Letters 2: 171-178.

Recibido el 12 de marzo de 2013 y aceptado el 7 de junio de 2013

Editor: Claudia Bustos 